\title{
A REVIEW: STATUS OF GENETIC MODULATED NONSMALL CELL LUNG CANCER TARGETS AND TREATMENT (CURRENT UPDATES IN DRUGS FOR NON-SMALL CELL LUNG CANCER TREATMENT)
}

\author{
SATYENDER KUMAR ${ }^{1 *}$, PRIYANK PUROHIT ${ }^{1}$, SEEMA DAGAR ${ }^{2}$
}

${ }^{1}$ Department of Pharmacy, School of Medical and Allied Sciences, Galgotias University, 1-Knowledge Park, Phase II, Greater Noida - 201 306, Uttar Pradesh, India. ${ }^{2}$ Department of Pharmaceutical Administration and Management, Institute of Pharmaceutical Sciences, Kurukshetra University, Kurukshetra - 136 119, Haryana, India. Email: sjinagal@gmail.com

Received: 27 March 2018, Revised and Accepted: 07 May 2018

ABSTRACT

Genetic modifications or mutations has been a bottleneck for the treatment of cancer; it is widely known to play a vital role in the progression of metastatic level/stage within the nonsmall cell lung cancer (NSCLC). The NSCLC of cancer is responsible for lung cancer lawsuits. In the various genetic mutations related study has been concluded with the various genes findings, which are named as the epidermal growth factor receptor, anaplastic lymphoma kinase, Kristen rat sarcoma virus, ROS proto-oncogene 1, human epidermal growth factor, B-RAF proto-oncogene, rearranged during Transfection, MET, Phosphatidyl 3-kinases CA, IGF-1R, NTRK1, FGFR1, and DDR2. The various research data supported this study. The involvement of the gene in the NSCLC patients made a paradigm shift in the drug discovery. The presence of one mutation in connection with some other could have an impact on NSCLC remedy.Utilizing this genotype-directed therapy for an advanced NSCLC has to turn out to be an appealing and efficacious treatment strategy. Here in the advancement of research, related genetic modulated targets and treatment have been discussed, particular genetic mutations help to find new updated interventions or medicinal drugs for the treatment of NSCLC. In there view, we have comprehensively arranged the mutation type and treatment with the status of NSCLC.

Keywords: Non-small lung cancer, Cancer, Mutation, Gene, Lung cancer.

(c) 2018 The Authors. Published by Innovare Academic Sciences Pvt Ltd. This is an open access article under the CC BY license (http://creativecommons. org/licenses/by/4. 0/) DOI: http://dx.doi.org/10.22159/ajpcr.2018.v11i8.26271

\section{INTRODUCTION}

\section{Introduction: Lung cancer}

Lung cancer is the categorically found in lung tissues, mainly in the air passages of cells lining. Lung cancer conventionally categorized into two types as follows: (1) Small cell lung cancer (SCLC) and (2) nonSCLC (NSCLC) [1]. Among the all lung cancers, approximately $85 \%$ are recognized as nonsmall cell, and approximately $75 \%$ of these are metastatic, or advanced, at diagnosis level [2,3]; however, SCLC, also known as oat cell cancer and approximately (10-15\%) of all lung cancers. The major difference in between SCLC and NSCLC can be identified under easily by the simple microscopy technique. The SCLC cells appear small and originally packed with the nucleus (cell control center) [4]. NSCLC is further categorized in adenocarcinoma, large cell carcinoma and squamous cell carcinoma, which are grouped collectively due to the fact they behave in a similar manner and respond to remedy in a similar way [4-6]. Common signs and symptoms in NSCLC including chest pain, hoarseness, weight loss, cough, shortness of breath, bone ache, yellowing of skin and eyes, and lumps close to the surface of the body [4].

In the last year (2017) report about lung cancer gave the surprising result about the case of lung cancer, it revealed that the number of new cases 222,500 of lung cancer and 155,870 deaths from lung cancer predicted by the American Cancer Society in the United States [3,4]. Lung cancer is the leading cause of most cancers loss of life among all type of cancers. Among the all types of genetically define people affected from lung cancer is more than other type named as colon, breast, and prostate cancers [4,7]. Survival rate varies by gender and cancer stage at the time of diagnosis. From the report of the surveillance, epidemiology, and results program, the relative survival rate of 5 years is $15 \%$ for men and $21 \%$ for women and the 5-year survival rate of NSCLC patients in different stages, stage IA (49\%), stage IB (45\%), stage IIA (30\%), stage IIB (31\%), stage IIIA (14\%), stage IIIB (5\%), and metastatic or stage IV (1\%) [4] at different tumor stages, the mechanism of NSCLC progression may be different [8] and specific. The genetic mutation helps to identify better target drug treatment for NSCLC patients $[5,8]$. In almost all the cases of malignancy, these genetic modifications are acquired during a person's lifetime and are available in specific cells in the lung. These mutations, can be referred as somatic mutations, which are not inherited. Somatic mutations in lots of unique genes have been located in lung malignancy cells. Cancer occurs due to the genetic mutations in vital genes, specifically those that manipulate cell development and division or DNA damage repair. These modifications/ mutations enable cells to develop and divide widely to form a tumor [9].

\section{Genetic modifications in NSCLC}

Lung cancer is a major type among other many distinct subsets of cancer that can classify by way of numerous factors, including histology and the molecular make-up of a tumor $[2,10]$. Genetic changes in lung cancer disease caused by certain changes to genes that decide that how our cells grow and divide. The genetic changes include DNA mutations, which affect the genetic functions or molecular mechanisms known as molecular abnormalities. The molecular abnormalities are associated with growthpromoting and genetic alterations or growth-promoting genes [11,12].

\section{Genes, chromosomes, and DNA}

Smoking is the major cause of lung cancer among the other risk factors. However, $25 \%$ of lung cancer patients are nonsmokers. Various reported a study of different molecular signature, etiology, histology, and location of NSCLC for smokers and nonsmokers [10]. Adenocarcinoma was found prevalent in nonsmoker NSCLC patients. There are certain genes and chromosomes that have a connection with an increased risk of lung cancer at the molecular level. Carriers of TP53 germline sequence variations which also prone for a smoker than three times more likely to develop lung cancer than nonsmokers. There are several reports for a marker on chromosome 15 associated with lung cancer. Our genes made up by DNA, which controls cells functions mostly the cell division process. However, DNA also can influence the risk for the development of certain diseases, such as different type of cancers. The DNA change appears in 
the people who inherit a particular chromosome (chromosome 6) are more likely to develop lung cancer [5], but some genes such as oncogenes, which control cell growth, including division of new cells, are thought to be important in the development of NSCLC $[4,13]$. The identification and increased understanding of molecular abnormalities in lung cancer research is mainly involve in identifying of molecular targets including HER family of receptors, anaplastic lymphoma kinase (ALK), phosphatidylinositol 3-kinase (PI3K)/RAC-alpha serine/threonineprotein kinase $(\mathrm{AKT}) / \mathrm{mTOR}$, epidermal growth factor receptor (EGFR), Kristen rat sarcoma virus (KRAS), IGF-1R, MET, and ROS [2,12]. The frequency of genetic alterations in NSCLC such as the oncogene KRAS mutated in approximately 30\% of lung cancer cases; FGFR-1 amplification (20\%); ALK rearrangement (3-7\%); AKT1 mutation (1\%); DDR2 mutation (approximately 4\%); human epidermal growth factor (HER2) mutation (2-4\%); KRAS mutation (12-25\%); MEK1 mutation (1\%); NRAS mutation (1\%); Phosphatidyl 3-kinases (PIK3) CA mutation (1-3\%); PTEN mutation (4-8\%); ROS proto-oncogene 1 (ROS1) rearrangement (1\%); MYC (2.5-10\%); Cyclin D1 (5\%); EGFR mutation (10-35\%); rearranged during transfection (RET) rearrangement (1\%); and C-erbB2 (Her-2/neu) or BCL2 overexpression involved in approximately $25 \%$ of cases; MET amplification and B-RAF protooncogene (BRAF) mutations present in about 1-3\% of NSCLC $[11,14]$. This information was utilized to develop targeted therapies [2,10] for the treatment of NSCLC. Molecular mechanism of oncogenes including EGFR, AKT1, BRAF, HER2, KRAS, MEK1, MET, NRAS, PIK3CA, RET, and ROS1 are currently available targets, and the updation of drugs and the clinical trials status has been discussed.

\section{EGFR}

EGFR belongs to receptor tyrosine kinases (RTKs) $[10,15]$ family that includes EGFR/ERBB1, HER2/ERBB2/NEU, HER3/ERBB3, and HER4/ ERBB4. The binding of growth factors or ligands, such as EGF, trigger a conformational change that facilitates homodimer or heterodimer receptor formation, thus resulting in activation of EGFR tyrosine kinase activity. Activated EGFR phosphorylates and its substrates, resulted in multiple downstream pathways activation within the cell The activation process involve the cell proliferation process by PI3K-AKT-mTOR, RASRAF-MEK-ERKor MAPK and the PI3K-AKT-mTOR pathways, which involved in cell proliferation (Fig. 1) [16,17].

\section{EGFR role in lung cancer}

EGFR deregulation (62\%) observed in NSCLC ( $~ 50 \%$ of non-smokers and $5-15 \%$ smokers). EGFR mutations is most commonly found in various cancers subtypes including NSCLC in which it occur within its 4 exons (18-21), which encodes a portion of the Tyrosine kinase domain (Fig 2). Heterozygous mutations most prominent in EGFR, with the mutant allele, showing gene amplification and these point mutations occurred as exon 19 deletions or exon 21 L858R point mutations accountable for $44 \%$ and 41\% EGFR-TK mutations, respectively. These mutations increase EGFR activity, leading to hyperactivity of downstream signalling pathways [18].

\section{EGFR inhibitors used in NSCLC with EGFR gene mutations}

Since EGFR dysregulation most common in NSCLC, small molecule Tyrosine Kinase Inhibitors,Erlotinib (Tarceva ${ }^{\circledR}$, Roche); Gefitinib (Iressa ${ }^{\circledR}$, AstraZeneca) and monoclonal antibodies (mAb) - Cetuximab (Erbitux ${ }^{\circledR}$, Merck) [19] target the EGFR-TK activity and used in the treatment of EGFR mutation [17]. Erlotinib (Tarceva); afatinib (Gilotrif); gefitinib (Iressa), D761 and T854A can be used as a first-line treatment for advanced NSCLCs with EGFR gene mutations [17]. Osimertininb (Tagrisso) targets T790 mutation (methionine substitution for threonine) in axon 20 of EGFR gene which hinder the interaction of inhibitor with receptor [5,20]. Cetuximab and necitumumab (Portrazza), monoclonal antibodies that also target EGFR.

\section{Treatments available for EGFR mutations}

First generations EGFR tyrosine kinase inhibitors

The mechanism of action for EGFR-TKIs (first-generation): Blockage of the downstream signaling activation induced by EGFR through ATPbinding sites binding $[15,21,22]$.

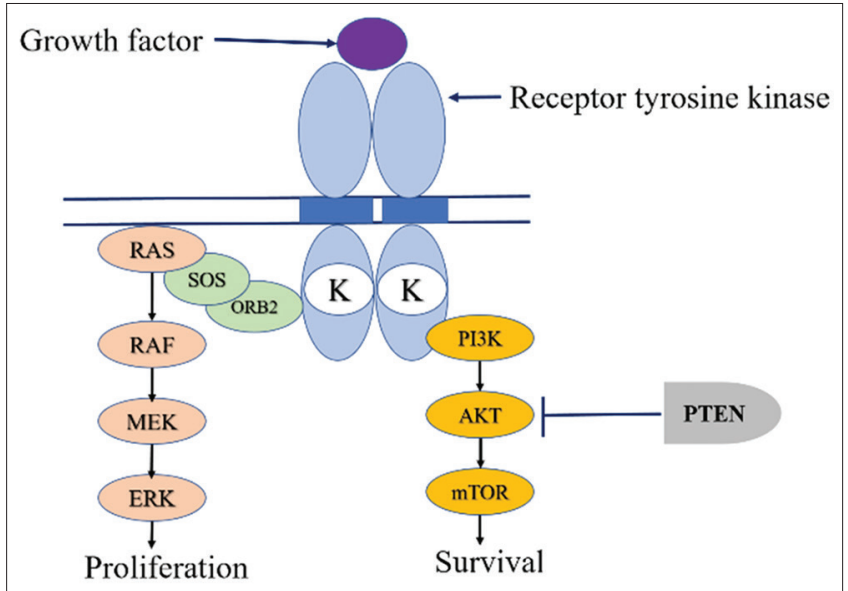

Fig. 1: Schematic diagram of EGFR signaling pathway [16]. Growth factor binding to EGFR results in activation of the MAPK signaling pathway (RAS-RAF-MEK-ERK) and the phosphatidylinositol 3-kinase (PI3K) pathway (PI3K-AKT-mTOR). The letter "K" within the schema denotes the tyrosine kinase domain

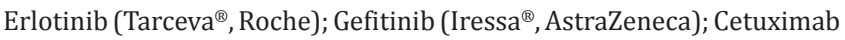
$\left(\right.$ Erbitux $^{\circledR}$, Merck) $[19,23]$

Erlotinib (Tarceva ${ }^{\circledR}$, Roche): Erlotinib is reversibly EGFR-TKI and approved by Federal agency FDA as a first-line treatment for EGFR mutation in NSCLC patients mutation in 2013 [15,21].

Gefitinib (Iressa ${ }^{\circledR}$, AstraZeneca): Gefitinib is taken into account as an oral first-generation EGFR TKI and approval by FDAas a first-line treatment for NSCLC patients with EGFR mutations in 2015 [15,21,24].

Cetuximab (Erbitux ${ }^{\circledR}$, Merck): Cetuximab is a chimeric monoclonal antibody that is directly acts on the EGFR mutation in NSCLC $[15,20,25,26]$.

Icotinib: Icotinib a Chinese drug and approved as selective EGFR-TKI in NSCLC [15].

\section{Second generation EGFR TK inhibitors}

The mechanism of action for EGFR TKIs (second-generation) is for the alteration of the non-heritable resistance that comes from the failure offirstgeneration EGFR TKIs. Therefore, the operating mechanisms of secondgeneration EGFR TKIs do not seem to be precisely like first-generation EGFR TKIs [15], for example, Afatinib (Giotrif ${ }^{\circledR}$, BoehringerIngelheim); Dacomitinib (PF-0299804, Pfizer); Neratinib (HKI-272, Pfizer); Pelitinib (EKB-569, Wyeth/Pfizer); Canertinib (CI-1033, Pfizer) [19].

Afatinib (Giotrif ${ }^{\circledR}$, BoehringerIngelheim): Afatinib is an anilinequinazoline derivative with a reactive acrylamide group which can modify the catalytic domains of EGFR, HER2, and ErbB-4, with orally bioavailable irreversible EGFR inhibitor and afatinibaction may longer than reversible EGFR TKIs [15,27].

Dacomitinib (PF-0299804, Pfizer): Dacomitinib is EGFR-TKI in NSCLC by targeting ErbB2 and ErbB4 kinase [15].

Neratinib (HKI-272, Pfizer):Neratinib is an irreversible pan-ErbB inhibitors and found with positive response in NSCLC patients with G719X mutations in EGFR [28].

Pelitinib (EKB-569, Wyeth/Pfizer):Pelitinib is a potent irreversible EGFR-TKI and currently in clinical trials for the NSCLC treatment [28].

Canertinib (CI-1033, Pfizer):Canertinib is 3-chloro,4-fluoro,4anilinoquinazoline and an irreversible inhibitor for TKI domain of the erbB receptors [29]. 


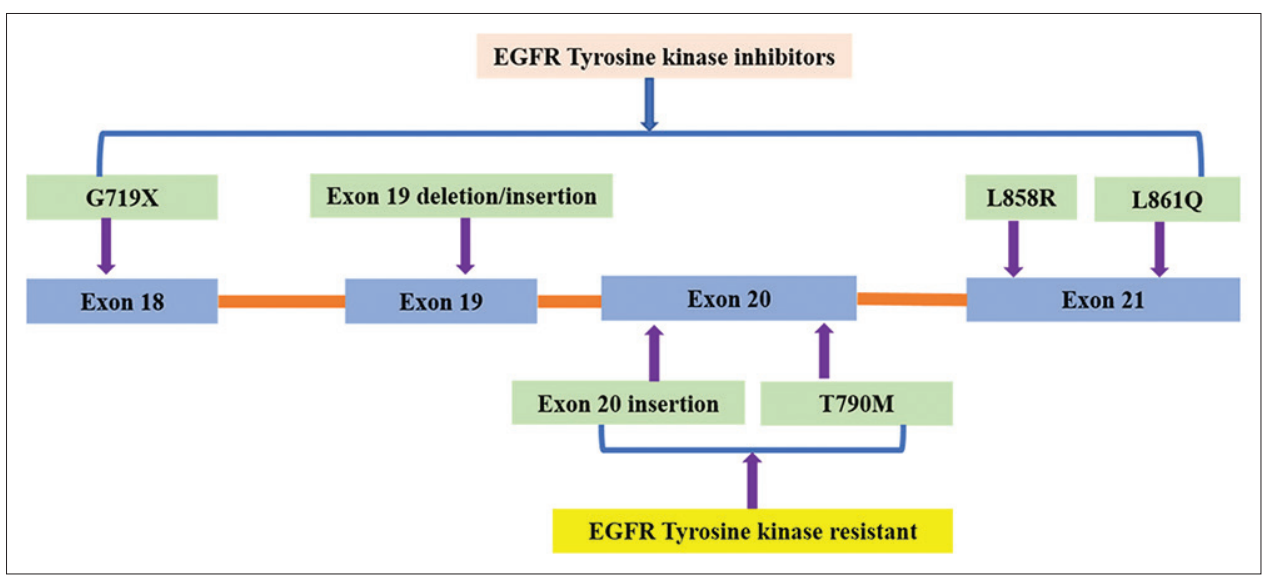

Fig. 2: Schematic representation of EGFR genetic mutations [10,16]. Exons 18-21 of the EGFR kinase domain are depicted. Mutations above the schematic are associated with sensitivity to EGFR TKIs. Mutations listed below the schematic are associated with EGFR TKI resistance.

\section{Third generations EGFR TK inhibitors}

Nowadays, many EGFR TKIs used in the treatment of NSCLC patients. Unfortunately, all patients treated with gefitinib, erlotinib, or afatinib produces resistance. To an outsized extent, either non-inheritable or intrinsic resistance reduced the effectualness of EGFR TKIs. These reported mechanisms embody the event of T790M mutation, CMET amplification, HER2 amplification, microscopic anatomy transformation to small-cell microscopic anatomy. Among them, the foremost vital mechanism of non-inheritable resistance is the EGFR T790M mutation. It had been according that prevalence frequency of T790M mutation was from $49 \%$ to $63 \%$ when rebiopsies. To beat this new issue,completely difference third-generation EGFR-mutant-selective TKIs, similar to osimertinib, rociletinib, and olmutinib, appeared. These agents square measure specifically designed to inhibit EGFR T790M while not wildtype EGFR, that structurally completely different from first-generation and second-generation inhibitors [15,30-32]

Osimertinib (taggriso, AZD9291): Osimertinibis an irreversible and selective inhibitor for both T790M mutation-positive EGFR and sensitizing EGFR mutation in NSCLC [15]

C01686 (rociletinib): C01686 is also third-generation irreversible EGFR-TKI in NSCLC [15].

AC0010: AC0010 is an irreversible inhibitor of EGFR mutants in NSCLC and based on pyrrolopyrimidine [15].

HM61713 (olmutinib):HM61713 is a selective inhibitor for T790M EGFR mutants except EGFR wild-type in NSCLC [15].

\section{Fourth-generation EGFR TKI's}

In $32 \%$ of patients, the new EGFR C797S mutation occurring. Hence, the fourth-generation EGFR-TKIs appeared to overcome this mutation $[15,31,33]$. for example, EAI045.

EAI045: EAI045 only works in the combination with cetuximab and targets T790M and C797S EGFR mutations [15].

\section{Clinical trials on EGFR in NSCLC}

In the USA, 93 clinical trials including Phase-I (NCT02639091; NCT02637531; NCT02608385; NCT02595866; NCT02520778; NCT02563548; NCT02496663; NCT02475213; NCT02409108; NCT02381314; NCT02365662; NCT02364609; NCT02327468; NCT02309892NCT02309177; $\quad$ NCT02157792; $\quad$ NCT02143466; NCT02113813; NCT00889954; NCT02298153; NCT02071862; NCT02013219; NCT01999985), Phase II (NCT02132598; NCT02323126; NCT02314364; NCT02312622; NCT02045446;
NCT01857271; NCT01854034; NCT01829217; NCT01702844; NCT01746251; NCT01620190; NCT01573702; NCT01553942; NCT01248247); Phase II and III (NCT02438722); Phase III (NCT02409342; NCT02193282); and no phase specified phases (NCT01294280; NCT02299141; NCT02194738; NCT02450591) and 33 clinical trials going on in Phase I (NCT02358473; NCT01998126; NCT01967095; NCT01966445; NCT02088112; NCT01839955; NCT02206763; NCT02192541; NCT02191891); Phase I and II (NCT02155465; NCT02580708); Phase II (NCT02485652; NCT02454842; NCT02448303; NCT02318368; NCT01532089; NCT01336634; NCT01935947; NCT01928160; NCT01877083; NCT02119650; NCT02289833; NCT02271906; NCT01306045); Phase III (NCT02453282; NCT02352948; NCT02322281; NCT02296125; NCT02066636; NCT02134015); Phase IV (NCT02151149); no specified phase (NCT01416688; NCT00900328) at unknown locations for EGFR in NSCLC as a target is mentioned in Tables 1 and 2 [16].

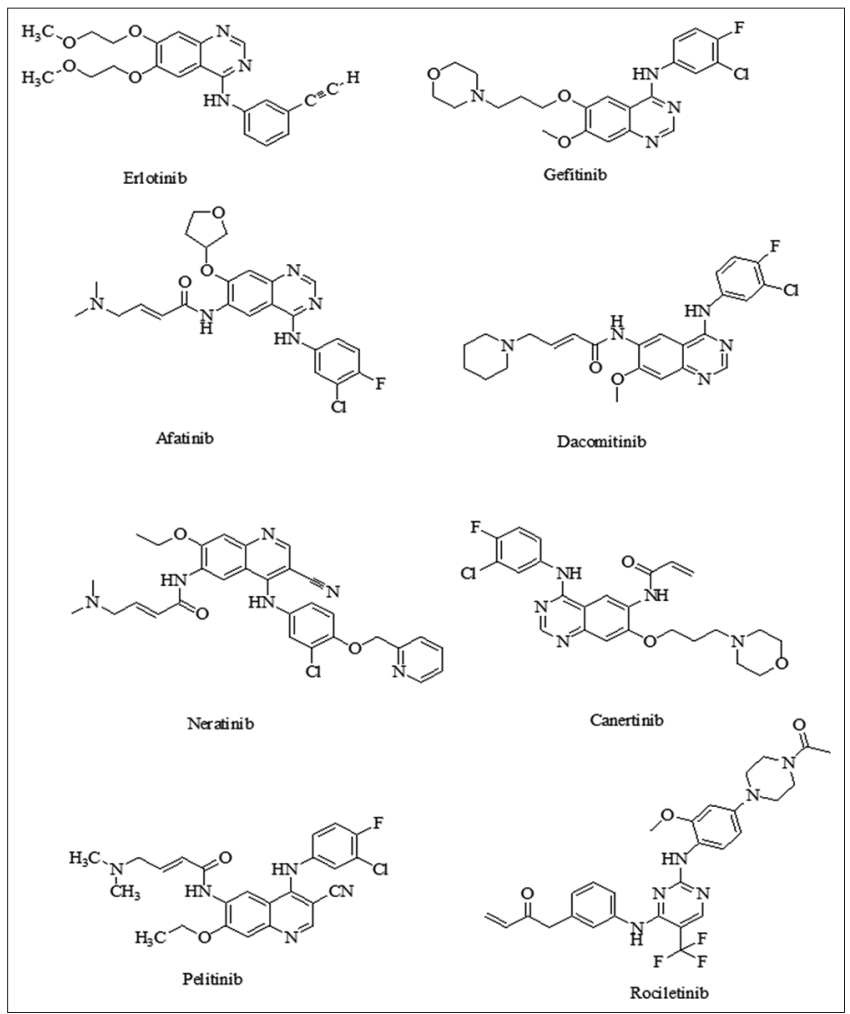


Table 1: Clinical trials on EGFR in NSCLC in the USA as mentioned below [16]

\begin{tabular}{|c|c|c|c|}
\hline Phase & Drug candidate & Protocol id & Current status \\
\hline \multirow[t]{20}{*}{ Phase I } & BAY 94-9343, pemetrexed and cisplatin & NCT02639091 & Recruiting \\
\hline & IP1-549 and nivolumab & NCT02637531 & Recruiting \\
\hline & Stereotactic body radiation therapy+pembrolizumab & NCT02608385 & Recruiting \\
\hline & Pembrolizumab & NCT02595866 & Recruiting \\
\hline & Osimertinib and navitoclax & NCT02520778 & Recruiting \\
\hline & Pembrolizumab with PEDPH-20 & NCT02563548 & Recruiting \\
\hline & Necitumumab with EGFR inhibitor AZD9291 & NCT02496663 & Recruiting \\
\hline & Exotherm total-body hyperthermia & NCT02409108 & Recruiting \\
\hline & Enoblituzumab (MGA271)+ipilimumab & NCT02381314 & Recruiting \\
\hline & ABBV-221 & NCT02365662 & Active, not recruiting \\
\hline & Pembrolizumab and afatinib dimaleate & NCT02364609 & Recruiting \\
\hline & INO-1400 vaccine+INO-9012 & NCT02327468 & Recruiting \\
\hline & l-DOS47 & NCT02309892 & Recruiting \\
\hline & Nab-paclitaxel/carboplatin & NCT02309177 & Active, not recruiting \\
\hline & VX-970 & NCT02157792 & Active, not recruiting \\
\hline & Autologous cytotoxic t-lymphocytes & NCT00889954 & Active, not recruiting \\
\hline & Atezolizumab (MPDL280A)+epacadostat (INCB024360) & NCT02298153 & Terminated \\
\hline & Glutaminase inhibitor cb-839 & NCT02071862 & Recruiting \\
\hline & Atezolizumab+erlotinib or alectinib & NCT02013219 & Active, not recruiting \\
\hline & Afatinib dimaleate and dasatinib & NCT01999985 & Active, not recruiting \\
\hline \multirow[t]{12}{*}{ Phase II } & Cabozantinib-s-malate & NCT02132598 & Recruiting \\
\hline & Nivolumab+EGF816 and nivolumab+INC280 & NCT02323126 & Recruiting \\
\hline & Stereotactic body radiation therapy & NCT02314364 & Recruiting \\
\hline & Pegylated irinotecan NKTR-102 & NCT02312622 & Recruiting \\
\hline & $\begin{array}{l}\text { Maintenance chemotherapy with or without stereotactic } \\
\text { body radiation therapy }\end{array}$ & NCT02045446 & Active, not recruiting \\
\hline & Erlotinib hydrochloride before surgery & NCT01857271 & Recruiting \\
\hline & HSP90 inhibitor AUY922 & NCT01854034 & Completed \\
\hline & Sunitinib malate & NCT01829217 & Active, not recruiting \\
\hline & Paclitaxel albumin-stabilized formulation & NCT01702844 & Active, not recruiting \\
\hline & Afatinib dimaleate & NCT01746251 & Active, not recruiting \\
\hline & $\begin{array}{l}\text { Afatinib dimaleate, combination chemotherapy, and } \\
\text { radiation therapy }\end{array}$ & NCT01553942 & Recruiting \\
\hline & $\begin{array}{l}\text { Erlotinib hydrochloride, AKT inhibitor MK2206, } \\
\text { selumetinib, or sorafenib tosylate }\end{array}$ & NCT01248247 & Active, not recruiting \\
\hline Phase II, Phase III & Afatinib dimaleate with or without cetuximab & NCT02438722 & Recruiting \\
\hline \multirow[t]{2}{*}{ Phase III } & $\begin{array}{l}\text { Aatezolizumab (MPDL3280A) compared with cisplatin or } \\
\text { carboplatin+pemetrexed or gemcitabine }\end{array}$ & NCT02409342 & Recruiting \\
\hline & Erlotinib hydrochloride & NCT02193282 & Recruiting \\
\hline \multirow[t]{4}{*}{ No phase specified } & Adjuvant chemotherapy & NCT01294280 & Recruiting \\
\hline & Nintedanib & NCT02299141 & Recruiting \\
\hline & Surgery & NCT02194738 & Recruiting \\
\hline & Local therapy and erlotinib hydrochloride & NCT02450591 & Completed \\
\hline
\end{tabular}

NSCLC: Non-small cell lung cancer, AKT1: RAC alpha serine/threonine-protein kinase, HSP90: Heat shock protein-90

Table 2: Clinical trials going on at unknown locations for EGFR in NSCLC as mentioned below [16]

\begin{tabular}{|c|c|c|c|}
\hline Phase & Drug candidate & Protocol id & Current status \\
\hline \multirow[t]{8}{*}{ Phase I } & Mogamulizumab+docetaxel & NCT02358473 & Completed \\
\hline & $\begin{array}{l}\text { Immune checkpoint inhibitor and erlotinib } \\
\text { hydrochloride or crizotinib }\end{array}$ & NCT01998126 & Active, not recruiting \\
\hline & Low- and high-dose erlotinib hydrochloride & NCT01967095 & Active, not recruiting \\
\hline & GSK2849330 & NCT01966445 & Completed \\
\hline & MEDI4736 (anti pd-l1)+gefitinib & NCT02088112 & Active, not recruiting \\
\hline & Erlotinib hydrochloride and quinacrine dihydrochloride & NCT01839955 & Completed \\
\hline & Ganetespib and ZIV-aflibercept & NCT02192541 & Terminated \\
\hline & BI 836845 plus afatinib & NCT02191891 & Active, not recruiting \\
\hline Phase I, & Ruxolitinib phosphate and erlotinib hydrochloride & NCT02155465 & Completed \\
\hline Phase II & Rociletinib+trametinib & NCT02580708 & Completed \\
\hline \multirow[t]{4}{*}{ Phase II } & HM61713 & NCT02485652 & Active, not recruiting \\
\hline & Pembrolizumab and acalabrutinib & NCT02448303 & Active, not recruiting \\
\hline & Ficlatuzumab plus erlotinib versus placebo plus erlotinib & NCT02318368 & Terminated \\
\hline & Erlotinib hydrochloride with or without bevacizumab & NCT01532089 & Active, not recruiting \\
\hline
\end{tabular}


Table 2: (Continued)

\begin{tabular}{|c|c|c|c|}
\hline Phase & Drug candidate & Protocol id & Current status \\
\hline \multirow{11}{*}{ Phase III } & Azacitidine and entinostat & NCT01935947 & Terminated \\
\hline & $\begin{array}{l}\text { Pemetrexed disodium and carboplatin or } \\
\text { cisplatin } \pm \text { erlotinib hydrochloride or gefitinib }\end{array}$ & NCT01928160 & Withdrawn \\
\hline & Lenvatinib (E7080) & NCT01877083 & Active, not recruiting \\
\hline & Ruxolitinib+pemetrexed/cisplatin & NCT02119650 & Terminated \\
\hline & Trastuzumab emtansine & NCT02289833 & Active, not recruiting \\
\hline & Afatinib dimaleate BIBW 2992 & NCT02271906 & Terminated \\
\hline & MEDI-4736 \pm tremelimumab, paclitaxel+carboplatin & NCT02453282 & Active, not recruiting \\
\hline & $\begin{array}{l}\text { MEDI- } 4736+\text { tremelimumab and } \\
\text { vinorelbine+gemcitabine }\end{array}$ & NCT02352948 & Active, not recruiting \\
\hline & $\begin{array}{l}\text { Rociletinib (CO-1686), rociletinib, pemetrexed or } \\
\text { gemcitabine or paclitaxel or docetaxel }\end{array}$ & NCT02322281 & Active, not recruiting \\
\hline & AZD9291 versus gefitinib or erlotinib & NCT02296125 & Active, not recruiting \\
\hline & Nivolumab & NCT02066636 & Active, not recruiting \\
\hline \multirow{3}{*}{$\begin{array}{l}\text { Phase IV } \\
\text { No phase } \\
\text { specified }\end{array}$} & Patritumab, erlotinib, placebo & NCT02134015 & Terminated \\
\hline & Cetuximab, panitumumab, or erlotinib hydrochloride & NCT01416688 & Active, not recruiting \\
\hline & Laboratory biomarker analysis & NCT00900328 & Completed \\
\hline
\end{tabular}

EGFR: Epidermal growth factor receptor, NSCLC: Non-small cell lung cancer

\section{AKT1}

AKT1 oncogenic mutations are non-overlapping with other mutations which found in NSCLC. AKT1 is related to the serine-threonine protein kinases family which also includes subtypes AKT2 and AKT3. AKT1 plays a key role in cell growth, angiogenesis, proliferation, and survival. The distinct roles of the AKT isoforms in NSCLC are largely unknown, but AKT1 also downregulates PI3K pathway (Fig. 1) [33-37]

\section{Treatments available for AKT1 mutations}

- Ipatasertib (GDC 0068), which targets all three isoforms of AKT [38,39].

- Uprosertib (GSK2141795), also another inhibitor of all AKT isoforms, and in combination with oral MEK1/2 inhibitor, it is showed better results in the trials, still it is unclear the role of drug for lung cancer patients [38].

- MK2206, an another allosteric AKT inhibitor, which potentiate erlotinib activity in NSCLC cell lines as well as resensitized cells which developed erlotinib resistance through the HGF-MET axis. In a Phase II trial, MK220 with erlotinib for patients with advanced NSCLC progressed after previous response to erlotinib (NCT01294306), In Phase-I trial, MK2206 and gefitinib in NSCLC patients also active (NCT01147211) [38]. MK-2206 in combination with erlotinib in NSCLC cell lines showed synergistic growth inhibition [40].

- Perifosine, is a phospholipid derivative of alkyl phosphocholine; in Phase I/II trials an oral AKT inhibitor studied for NSCLC (NCT00399789). Perifosine and radiation also recommended in NSCLC [40].

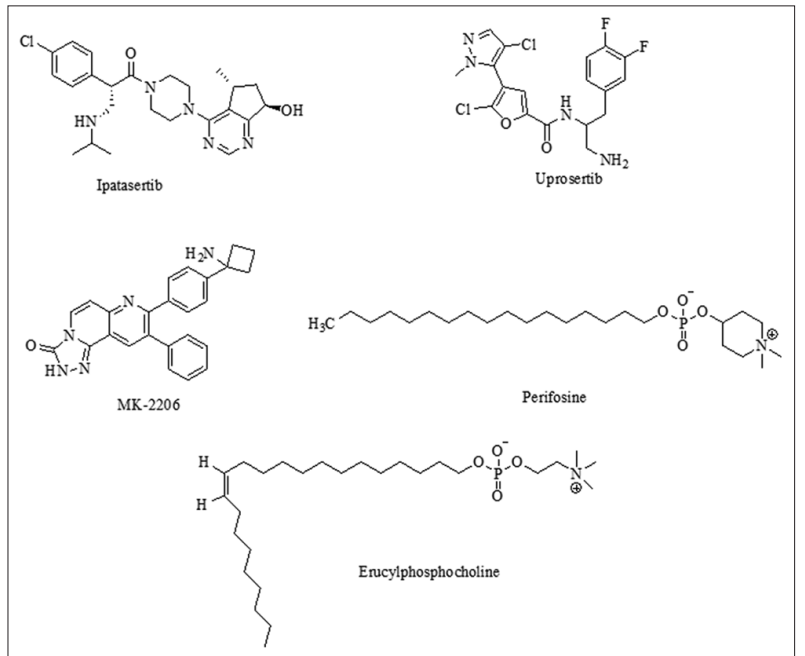

- RX-0201 (Archexin), is a nano-polymer Akt antisense oligonucleotideanticancer drug [40].

- Erucylphosphocholine, is structurally related to perifosine appears to inhibit Akt [40].

- $\quad$ PBI-05204, a derivative of Nerium oleander and an inhibitor of Akt [40].

- GSK690693, appeared to delay tumor growth irrespective of Akt activation status [40].

- XL-184, a dual inhibitor of Akt and p70S6K [40]

\section{Clinical trials on AKT1}

In the USA, two clinical trials including Phase II (NCT02642042; NCT01248247) and some clinical trials at unknown locations in Phase II (NCT02271906; NCT01306045) are going on AKT1 as a target mentioned in Tables 3 and 4 [35]

\section{BRAF}

BRAF belongs to a family of serine-threonine protein kinases that includes A-Raf Proto-Oncogene and C-Raf Proto-Oncogene. RAF kinases are significant mediators in the MAP kinase signaling cascade and exert their impact predominantly via phosphorylation and MEK activation. This occurs following the (hetero or homo) dimerization of the RAF molecules. As a part of the MAP kinase pathway, RAF is involved in many cellular approaches, along with differentiation, cell proliferation, and transcriptional regulation. BRAF mutation implicated in the pathogenesis of several cancers, including melanoma, NSCLC, colorectal cancer, papillary thyroid cancer, and ovarian cancer.BRAF mutations also observed in glioma and gastrointestinal stromal tumor (GIST) [41]. RAF receptor contains the regulative domain, associate in nursing activation loop, and a carboxyl terminus that contains the enzyme domain. The regulative domain is found among exons 1-10 within the amino $(\mathrm{N})$ terminus, whereas the enzyme domain is found among exons 11-18 at the carboxyl (C) terminus (Fig. 3) [17,42-46].

\section{Treatment available for BRAF inhibition}

- Vemurafenib, a selective BRAFV ${ }^{600}$ inhibitor in the treatment of progressive BRAF mutant NSCLC $[42,47]$

- Dabrafenib, is an another a selective BRAFV ${ }^{600}$ inhibitorin NSCLC and could represent a treatment option for a population of patients with limited therapeutic options $[42,48]$.

Clinical trials on BRAF

In the USA, nine clinical trials in Phase I NCT02595866; Phase II (NCT01827384); Phase I, Phase II (NCT02452424; NCT02437136; 
Table 3: Clinical trial on AKT1 in NSCLC as mentioned below [48]

\begin{tabular}{lll}
\hline Phase & Drug Candidate & Protocol id \\
\hline Phase II & $\begin{array}{l}\text { Docetaxel, laboratory biomarker analysis, } \\
\text { trametinib } \\
\text { Erlotinib hydrochloride, MK2206, selumetinib, } \\
\text { or sorafenib tosylate }\end{array}$ & NCT02642042 \\
& NCT01248247 \\
\hline
\end{tabular}

NSCLC: Non-small cell lung cancer, AKT1: RAC alpha serine/threonine-protein kinase

Table 4: Clinical trial on AKT1 in unknown locations in NSCLC as mentioned below [48]

\begin{tabular}{lll}
\hline Phase & Drug candidate & Protocol id \\
& & status \\
\hline Phase II & $\begin{array}{l}\text { Afatinib dimaleate before surgery BIBW 2992 } \\
\text { Targeted enzyme inhibitor therapy (AZD6244, MK-2206, } \\
\text { lapatinib) }\end{array}$ & NCT02271906 \\
& NCT01306045 & \\
\hline
\end{tabular}

NSCLC: Non-small cell lung cancer, AKT1: RAC alpha serine/threonine-protein kinase

NCT02428712; NCT02404441; NCT02327078; NCT02178722; NCT01325441) and some clinical trials at unknown locations in Phase I, Phase II (NCT01449058); Phase II (NCT01336634; NCT01306045) are going on BRAF as a target mentioned in Tables 5 and 6 [41].

\section{ALK}

It is responsible for $3-7 \%$ of all lung cancers $[5,49]$. For instance, activating missense mutations within full-length ALK are found in a subset of neuroblastomas [49]. The rearrangement in EML-4-ALK is the most common ALK rearrangement seen in NSCLC patients [5]. In nonsmoker lung cancer patients, EML-4 mutations are common or had been light smokers whose tumors lack both EGFR and KRAS mutations [5]. Using comparison, ALK fusions found in anaplastic large cell lymphoma (e.g., NPM-ALK, colorectal cancer, inflammatory myofibroblastic tumor). ALK fusions include the whole ALK tyrosine kinase domain. Finally, the ALK copy number and protein expression aberrations have also been found in rhabdomyosarcoma [49]. Other ALK mutations do not been found involving EML-4, including KIF5B-ALK and TFG ALK. Regarding treatment, patients suffered from EML4-ALK fusions or ALK rearrangements not benefited from EGFR-specific tyrosine kinase inhibitor therapy in NSCLC and ovarian cancer [49-52]

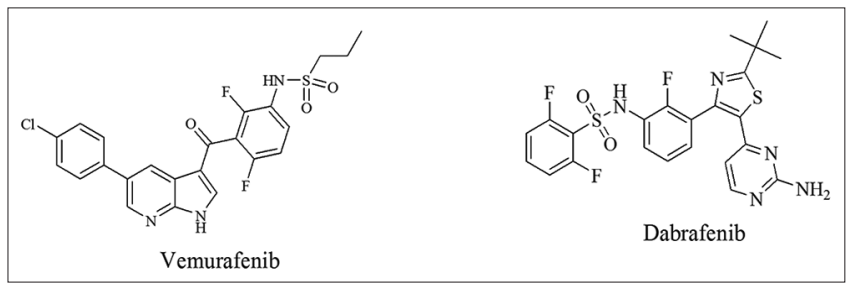

The N-terminal fusion partners (X) promote dimerization and growth factor binding potentiate ALK tyrosine kinase activity results in the cell growth, proliferation, and anti-apoptosis. Signaling downstream of ALK fusions results in the activation of cellular pathways known to be involved in cell growth and cell proliferation (Fig. 4) [49].

\section{Interventions for the treatment of ALK mutations}

ALK fusion oncogene associated with advanced NSCLC is highly sensitive to ALK tyrosine kinase (TK) inhibitors [51,53].

- Crizotinib, (Xalkori®, Pfizer), is indicated as second-line therapy and targets activated RTKs that result from EML4-ALK and other ALK fusions. Previously untreated advanced non-squamous ALK-positive NSCLCsufferers had been randomized to receive crizotinib $250 \mathrm{mg}$ by mouth twice a day $(\mathrm{n}=172)$. Intravenous chemotherapy(pemetrexed $500 \mathrm{mg}$ plus either cisplatin $75 \mathrm{mg}$ or carboplatin all administered intravenously every three weeks for $\leq 6$ cycles, $n=171$ ) [5].

- Ceritinib, is a second-generation ALK inhibitor and approximately 20 times more potent than Crizotinib [53]. FDA approved, ceritinib

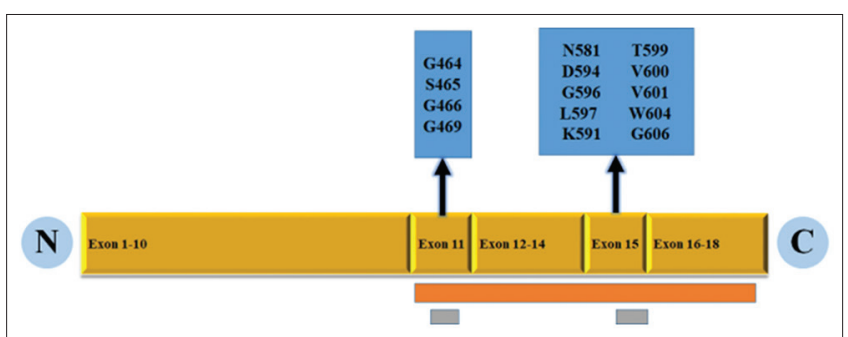

Fig. 3: BRAF mutations within the kinase domain (Red bar): In exons 11 and 15. The activation segment and conserved glycine motif (G-loop) (Grey bar): In exon 11 and in exon 15.

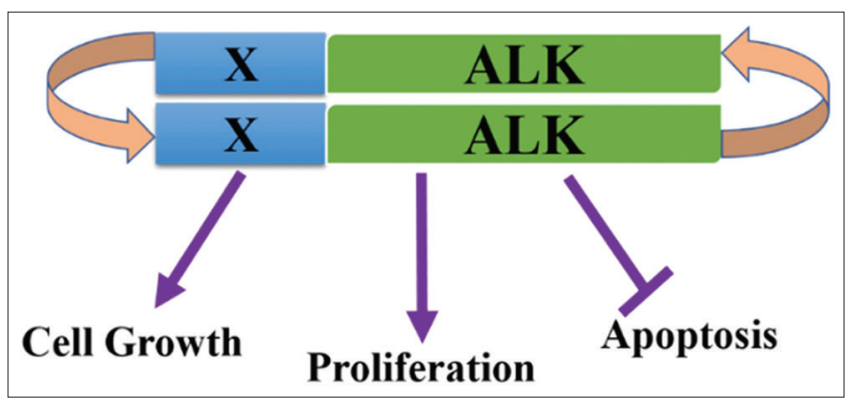

Fig. 4: Cell signaling pathway of ALK. Dimerization of the ALK fusion mediated by the fusion partner ("X"), results in constitutive activation of the ALK tyrosine kinase. ALK signaling results in pro-growth and anti-apoptosis.

for patients who have been intolerant of crizotinib. In clinical and preclinical studies, ceritinib showed activity against cells that were either sensitive or resistant to crizotinib and including with the most common L1196M and G1269A resistance mutations [53].

- Alectinib, is another second-generation ALK inhibitor that showed activity in crizotinib-resistant lung cancer along with brain cancer. It approved by FDA for the treatment of patients with an ALKpositive mutation in NSCLC patients who have shown intolerance for crizotinib [53].

- Brigatinib, is an under investigation for ALK inhibition that yet not approved for clinical use. However, brigatinib associated with early pulmonary toxicity in a small percentage of cases [53].

- Lorlatinib, is another investigational ALK inhibitor that has shown promising activity in a phase I study. Importantly, lorlatinib has proven past time in patients whose tumors harbor the highly resistant mutation ALK G1202R. This mutation confers resistance to different next-generation ALK inhibitors, including ceritinib, alectinib, and brigatinib [53] 
Table 5: In USA, clinical trial on BRAF in NSCLC as mentioned below [40]

\begin{tabular}{|c|c|c|c|}
\hline Phase & Drug candidate & Protocol id & Current status \\
\hline Phase I & Pembrolizumab and laboratory biomarker analysis & NCT02595866 & Recruiting \\
\hline Phase II & $\begin{array}{l}\text { Molecular profiling-based targeted therapy (adavosertib, } \\
\text { carboplatin, everolimus) }\end{array}$ & NCT01827384 & Suspended \\
\hline \multirow[t]{6}{*}{ Phase I, Phase II } & PLX3397+pembrolizumab & NCT02452424 & $\begin{array}{l}\text { Active, not } \\
\text { recruiting }\end{array}$ \\
\hline & Entinostat with pembrolizumab & NCT02437136 & Recruiting \\
\hline & PLX8394 & NCT02428712 & Recruiting \\
\hline & Nivolumab, epacadostat, chemotherapy & NCT02327078 & Recruiting \\
\hline & Pembrolizumab (MK-3475)+epacadostat (INCB024360) & NCT02178722 & Recruiting \\
\hline & BBI608, paclitaxel & NCT01325441 & Recruiting \\
\hline
\end{tabular}

NSCLC: Non-small cell lung cancer

Table 6: Some clinical trials on BRAF at unknown locations as mentioned below [40]

\begin{tabular}{lll}
\hline Phase & Drug candidate & Protocol id \\
\hline Phase I, Phase II & MEK162+BYL719 & NCT01449058 \\
Phase II & Dabrafenib monotherapy+trametinib & NCT01336634 \\
& Targeted enzyme inhibitor therapy (AZD6244, MK-2206, & NCT01306045 \\
& Lapatinib) & Active, not recruiting \\
\hline
\end{tabular}

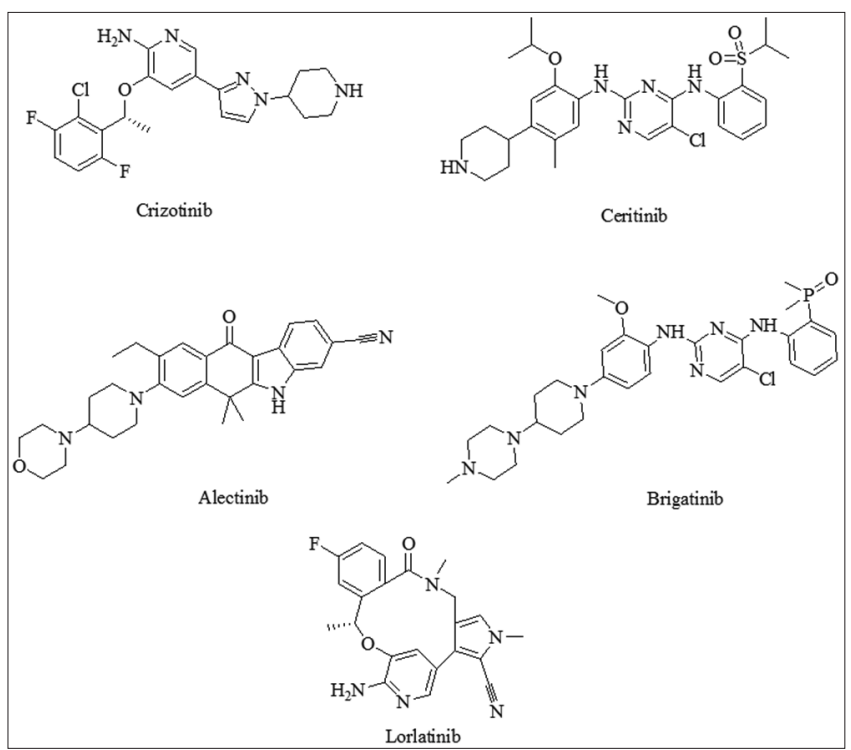

Clinical trials on ALK1

In the USA, 62 clinical trials including in Phase I (NCT02639091; NCT02637531; NCT02608385; NCT02595866; NCT02563548; NCT02422589; NCT02451930; NCT02511184; NCT02409108; NCT02393625; NCT02309892; NCT02321501; NCT02013219; NCT01730118; NCT01803282; NCT01772004; NCT02299505);Phase II (NCT02420314; NCT02414139; NCT02568267; NCT02513667; NCT02498613; NCT02591615; NCT02314364; NCT02312622; NCT02132598; $\quad$ NCT02045446; $\quad$ NCT01935336; NCT01928576; NCT01829217; NCT01822496; NCT01752400; NCT01228435; NCT01702844; NCT02336451; Phase I,II (NCT01970865; NCT01325441; NCT02584634; $\quad$ NCT02554812; $\quad$ NCT02523469; $\quad$ NCT02521051; NCT02487095; NCT02437136; NCT02408016; NCT02404441; NCT02403193; NCT02335918; NCT02327078; NCT02292550); Phase II \& III (NCT02154490); Phase III (NCT02504489; NCT02595944; NCT02657434; NCT02201992; NCT02409342; NCT02193282); no specified phase (NCT02178163; NCT02194738; NCT02299141) and some clinical trials in Phase I (NCT02358473; NCT02192541; NCT01998126; NCT01839955); Phase II (NCT01336634; NCT01306045; NCT01935947; NCT01877083); Phase III (NCT01828112); no specified Phase (NCT00900328) at unknown locations are going on ALK1 as a target mentioned in Tables 7 and 8 [49].

\section{ROS1}

ROS1 is related to a RTK of the insulin receptor family [54] that acts as a driver oncogene in 1-2\% of NSCLC through a genetic translocation between ROS1 and other genes, the most common of which is CD74 [54] chromosomal rearrangements regarding the ROS1 gene, on chromosome $6 \mathrm{q} 22$, were initially described in glioblastomas (e.g., ROS1). More recently, ROS1 fusions identified as a potential "driver" oncogene in NSCLC and cholangiocarcinoma [54]. ROS1 fusions incorporate a tyrosine kinase domain. Till date, those tested biologically possess oncogenic activity. Signaling downstream of ROS1 fusions outcomes in the activation of cellular pathways recognized too concerned with cell proliferation and cell growth (Fig. 1). ROS1 fusions are related to sensitivity in-vitro to tyrosine kinase inhibitors that inhibit ROS1 [54,55].

\section{Interventions for ROS1 inhibition}

- Crizotinib (xalkori): Is highly active and used as first line treatment or greater in patients with the advanced ROS1 arrangement of NSCLC $[56,57]$. Xalkori is the first and only FDA-approved treatment for patients with ROS-1 positive NSCLC $[57,58]$.

\section{Clinical trials on ROS1}

In the USA, eight clinical trials Phase I (NCT02637531; NCT02595866); Phase II (NCT02568267; NCT02426658; NCT02314364; NCT01702844; NCT01639508); Phase I, II (NCT01970865) and some clinical trials in Phase I (NCT01839955) at unknown locations are going on ROS1 as a target mentioned in Tables 9 and 10 [54].

\section{HER2}

It belongs to a RTKs family that includes EGFR/ERBB1, HER2/ERBB2/ NEU, HER4/ERBB4, and HER3/ERBB3. The gene for HER2 is placed on chromosome 17 and found to amplify with an increased copy number of several cancers. Amplification of HER2 has been discovered to promote tumorigenesis and to be involved in the pathogenesis of several human cancers. Until now, no ligand recognized for HER2. However, HER2 appears to be the preferred dimerization associate for all members of the ERBB family. The binding of ligand followed by HER2 heterodimerization outcomes in HER2 tyrosine kinase activation for activity. Then, activated HER2 phosphorylates its substrates, which lead to multiple downstream pathway activation, include PI3K-AKT-mTOR pathway and the RAS-RAF-MEK-ERK pathway, for involvement in cell proliferation, and cell survival, respectively (Fig. 5) [59]. 
Table 7: In USA, an around 62 clinical trials targeted as ALK in different phases mentioned below [48]

\begin{tabular}{|c|c|c|c|}
\hline Phase & Drug candidate & Protocol id & Current status \\
\hline \multirow[t]{15}{*}{ Phase I } & Anetumab ravtansine+pemetrexed and cisplatin & NCT02639091 & Completed \\
\hline & IPI-549 and nivolumab & NCT02637531 & Recruiting \\
\hline & Pembrolizumab and stereotactic body radiation therapy & NCT02608385 & Recruiting \\
\hline & Pembrolizumab and laboratory biomarker analysis & NCT02595866 & Recruiting \\
\hline & PEGPEM & NCT02563548 & Recruiting \\
\hline & Ceritinib+warfarin and midazolam & NCT02422589 & Active, not recruiting \\
\hline & Necitumumab (LY3012211) and pembrolizumab (MK3475) & NCT02451930 & Active, not recruiting \\
\hline & Exatherm total-body hyperthermia & NCT02409108 & Recruiting \\
\hline & Ceritinib+nivolumab & NCT02393625 & Recruiting \\
\hline & L-DOS47 & NCT02309892 & Recruiting \\
\hline & $\begin{array}{l}\text { Ceritinib (LDK378), ceritinib (LDK378) } 750 \text { mg, } \\
\text { everolimus }\end{array}$ & NCT02321501 & Recruiting \\
\hline & Atezolizumab+erlotinib or alectinib & NCT02013219 & Active, not recruiting \\
\hline & Autologous Ad HER2 dendritic cell vaccine & NCT01730118 & Recruiting \\
\hline & Andecaliximab, gemcitabine, nab-paclitaxel & NCT01803282 & Active, not recruiting \\
\hline & Ceritinib & NCT02299505 & Recruiting \\
\hline \multirow[t]{16}{*}{ Phase II } & Paclitaxel, carboplatin, ascorbic acid & NCT02420314 & Recruiting \\
\hline & INC280 (capmatinib) & NCT02414139 & Recruiting \\
\hline & Entrectinib (RXDX-101) & NCT02568267 & Recruiting \\
\hline & Ceritinib and stereotactic ablative radiation therapy & NCT02513667 & Recruiting \\
\hline & $\begin{array}{l}\text { Cediranib+olaparib and } 18 \mathrm{~F} \text {-fluoromisonidazole, cediranib maleate, } \\
\text { laboratory biomarker analysis }\end{array}$ & NCT02498613 & Recruiting \\
\hline & MK-3475, carboplatin, paclitaxel, pemetrexed & NCT02591615 & Recruiting \\
\hline & Stereotactic body radiation therapy & NCT02314364 & Recruiting \\
\hline & Pegylated irinotecan NKTR 102 and laboratory biomarker analysis & NCT02312622 & Recruiting \\
\hline & Cabozantinib-s-malate & NCT02132598 & Recruiting \\
\hline & Maintenance chemotherapy \pm stereotactic body radiation therapy & NCT02045446 & Active, not recruiting \\
\hline & Ponatinib hydrochloride & NCT01935336 & Recruiting \\
\hline & Azacitidine, entinostat, and nivolumab or nivolumab & NCT01928576 & Recruiting \\
\hline & Sunitinib malate & NCT01829217 & Active, not recruiting \\
\hline & Erlotinib hydrochloride or crizotinib and chemoradiation therapy & NCT01822496 & Active, not recruiting \\
\hline & Paclitaxel albumin-stabilized formulation & NCT01702844 & Active, not recruiting \\
\hline & LDK378 & NCT02336451 & Recruiting \\
\hline Phase I, & PF-06463922 and crizotinib & NCT01970865 & Active, not recruiting \\
\hline \multirow[t]{13}{*}{ Phase II } & BBI608 and paclitaxel & NCT01325441 & Recruiting \\
\hline & Avelumab+crizotinib or PF-06463922 & NCT02584634 & Recruiting \\
\hline & Avelumab+other cancer immunotherapies & NCT02554812 & Recruiting \\
\hline & Nivolumab with ALT-803 & NCT02523469 & Recruiting \\
\hline & Alectinib and bevacizumab & NCT02521051 & Recruiting \\
\hline & VX-970 and topotecan hydrochloride & NCT02487095 & Recruiting \\
\hline & Entinostat+pembrolizumab & NCT02437136 & Recruiting \\
\hline & Genetically modified t-cells & NCT02408016 & Active, not recruiting \\
\hline & PDR001 & NCT02404441 & Recruiting \\
\hline & PBF-509_80 mg, PBF-509_160 mg, PBF-509_320 mg and PDR-001 & NCT02403193 & Recruiting \\
\hline & Anti-cd27 (varlilumab) and Anti-pd-1 (nivolumab) & NCT02335918 & Active, not recruiting \\
\hline & Epacadostat+nivolumab & NCT02327078 & Recruiting \\
\hline & LEE011 and ceritinib & NCT02292550 & $\begin{array}{l}\text { Active, not } \\
\text { recruiting }\end{array}$ \\
\hline Phase II, & Biomarker-targeted second-line therapy & NCT02154490 & Recruiting \\
\hline Phase III & & & \\
\hline \multirow[t]{6}{*}{ Phase III } & Docetaxel+plinabulin versus docetaxel+placebo & NCT02504489 & Recruiting \\
\hline & Nivolumab after surgery & NCT02595944 & Recruiting \\
\hline & $\begin{array}{l}\text { Atezolizumab+carboplatin or cisplatin+pemetrexed vs carboplatin or } \\
\text { cisplatin+pemetrexed }\end{array}$ & NCT02657434 & Recruiting \\
\hline & Clinical observation, crizotinib, laboratory biomarker analysis & NCT02201992 & Recruiting \\
\hline & $\begin{array}{l}\text { Atezolizumab (MPDL3280A) versus platinum agent (cisplatin or } \\
\text { carboplatin)+(pemetrexed or gemcitabine) }\end{array}$ & NCT02409342 & Recruiting \\
\hline & $\begin{array}{l}\text { Erlotinib hydrochloride, clinical observation, laboratory biomarker analysis, } \\
\text { placebo }\end{array}$ & NCT02193282 & Recruiting \\
\hline \multirow{3}{*}{$\begin{array}{l}\text { No phase } \\
\text { specified }\end{array}$} & Cytology specimen collection procedure, laboratory biomarker analysis & NCT02178163 & Recruiting \\
\hline & Genetic testing & NCT02194738 & Recruiting \\
\hline & Nintedanib & NCT02299141 & Recruiting \\
\hline
\end{tabular}

ALK: Anaplastic lymphoma kinase 
Table 8: Some clinical trials targeted as ALK in different phases are going in an unknown location mentioned below [48]

\begin{tabular}{|c|c|c|c|}
\hline Phase & Drug candidate & Protocol id & Current status \\
\hline \multirow[t]{4}{*}{ Phase I } & Mogamulizumab+docetaxel & NCT02358473 & Completed \\
\hline & Ganetespib and ZIV-aflibercept & NCT02192541 & Terminated \\
\hline & $\begin{array}{l}\text { Immune checkpoint inhibitor and } \\
\text { erlotinib hydrochloride or crizotinib }\end{array}$ & NCT01998126 & Active, not recruiting \\
\hline & $\begin{array}{l}\text { Erlotinib hydrochloride and } \\
\text { quinacrine dihydrochloride }\end{array}$ & NCT01839955 & Completed \\
\hline \multirow[t]{3}{*}{ Phase II } & Dabrafenib and trametinib & NCT01336634 & Completed \\
\hline & $\begin{array}{l}\text { Azacitidine and entinostat before } \\
\text { chemotherapy }\end{array}$ & NCT01935947 & Terminated \\
\hline & Lenvatinib (E7080) & NCT01877083 & Active, not recruiting \\
\hline Phase III & Ceritinib, pemetrexed, docetaxel & NCT01828112 & Active, not recruiting \\
\hline No phase specified & Laboratory biomarker analysis & NCT00900328 & Completed \\
\hline
\end{tabular}

Table 9: In USA, clinical trials targeted as ROS1 in different phases are going on mentioned below [53]

\begin{tabular}{|c|c|c|c|}
\hline Phase & Drug candidate & Protocol id & Current status \\
\hline \multirow[t]{2}{*}{ Phase I } & IPI-549, nivolumab & NCT02637531 & Recruiting \\
\hline & Pembrolizumab and laboratory biomarker analysis & NCT02595866 & Recruiting \\
\hline \multirow[t]{5}{*}{ Phase II } & Entrectinib (RXDX-101) & NCT02568267 & Recruiting \\
\hline & Pemetrexed disodium & NCT02426658 & Recruiting \\
\hline & Stereotactic body radiation therapy & NCT02314364 & Recruiting \\
\hline & Paclitaxel & NCT01702844 & Active, not recruiting \\
\hline & Cabozantinib-s-malate & NCT01639508 & Recruiting \\
\hline
\end{tabular}

ROS1: ROS proto-oncogene 1

Table 10: Some clinical trials targeted as ROS1 in different phases at unknown locations mentioned below [53]

\begin{tabular}{lll}
\hline Phase & Drug candidate & Protocol id \\
& & Surrent \\
\hline Phase I & Erlotinib hydrochloride and quinacrine dihydrochloride & NCT01839955 \\
\hline
\end{tabular}

ROS1: ROS proto-oncogene 1

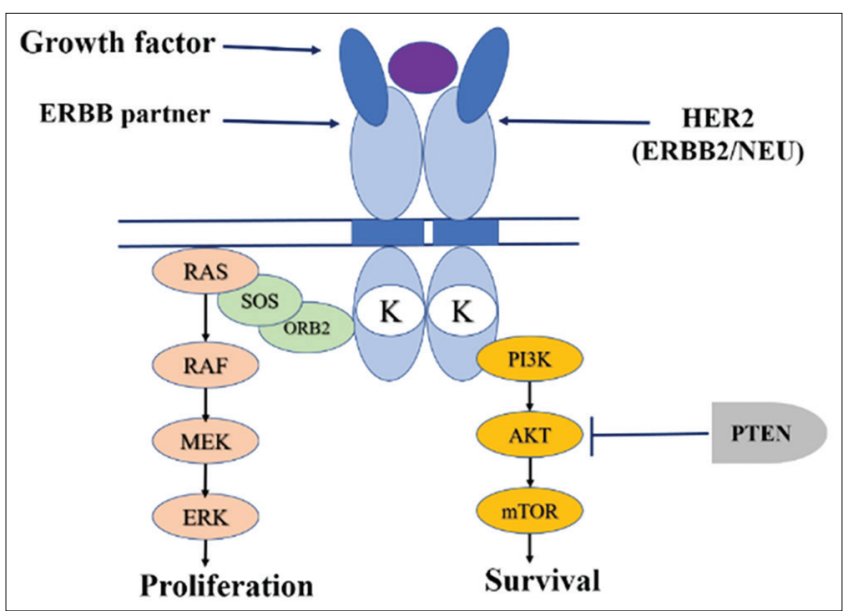

Fig. 5: Cell signaling of HER2. Growth factor binding to EGFR results in activation of the MAPK signaling pathway (RAS-RAF-MEK-ERK) and the phosphatidylinositol 3-kinase (PI3K) pathway (PI3K-AKT-mTOR). The letter "K" within the schema denotes the tyrosine kinase domain.

Interventions for HER2 mutations

- Lapatinib, tested in a phase II trial as an oral reversible dual TKI of EGFR and HER2 [60].

- Afatinib, evaluated in a phase II study, in which HER2 mutated five patients with advanced adenocarcinoma treated, 3 out of which were evaluable for response. Objective response observed in all three, even after the failure of other EGFR- and HER2-targeted treatments [60].
- $\quad$ Neratinib, is an irreversible pan ErbB-receptor family blocker, has been evaluated in a phase I trial in combination with temsirolimus by preclinical data suggesting synergy of HER2 inhibition and mTOR inhibition in lung cancer models [60]. It has also studied in a phase II trial with advanced NSCLC followed by erlotinib or gefitinib. Response rates in subgroups, including EGFR mutant, wild-type EGFR and EGFR TKI naive- adenocarcinoma with a light smoking history compared and results found 3.4\%, $0 \%$ and $0 \%$, respectively. Only a small subgroup of patients benefited from neratinib and based on these results; neratinib is no longer in development for NSCLC [61].

- Dacomitinib, is an irreversible pan-HER TKI, and it tested in a phase II cohort of patients with HER2-mutant or amplified lung cancers. An overall response rate in the 26 HER2-mutant patients was 13\%, and no response rate in four patients with HER2 amplification or the 2 with HER2 point mutations observed with Dacomitinib treatment [60]. Preliminary data of study in the HER2-mutant cohort reveal a partial response rate and $27 \%$ of the patients have maintained stable disease to date [61].

- Trastuzumab, not exhibited significant clinical activity in a phase II trial performed by the Cancer and Leukemia Group B as a singleagent against HER2 2+ or 3+ non-small cell lung carcinoma. However, randomized phase II trial investigated the addition of trastuzumab to gemcitabine and cisplatin, in 103 previously untreated HER2positive NSCLC patients. Trastuzumab was given both concomitantly with chemotherapy and as maintenance. Although the combination well tolerated, it failed to show a survival benefit in all HER2 IHCpositive lung cancer overall. The Eastern Cooperative Oncology Group, evaluated the combination of carboplatin, paclitaxel and trastuzumab in patients with HER2-positive $(1+$ to $3+)$ NSCLC in a phase II study and overall survival was found to be like historical 
data using carboplatin and paclitaxel alone, while patients with $3+$ HER2 expression did well in contrast to historical data [60].

- Pertuzumab, is a humanized monoclonal anti-HER2 antibody which is also first-in-class HER2 dimerization inhibitor, and that prevents HER2 dimerization and inhibits HER2 signaling. In a phase II trial, chronic NSCLC patients showed no response in 43 patients after pertuzumab monotherapy, but information on the mutational status of HER2 in these patients is lacking [60]

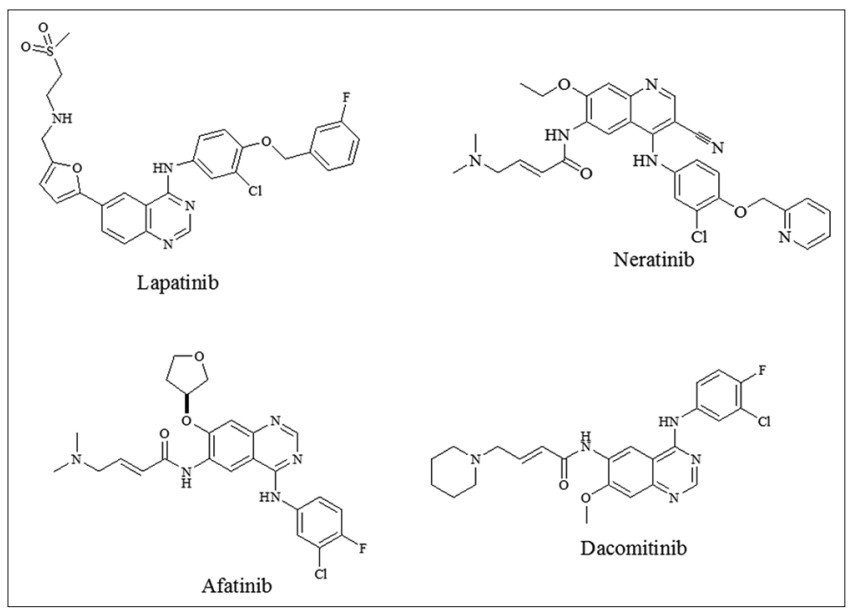

Clinical trials on HER2

In the USA, 16 clinical trials in Phase II (NCT02482311; NCT02327468; NCT02309177; NCT02157792; $\quad$ NCT02482311; NCT02327468; NCT02309177); Phase II (NCT02498613); Phase I, II (NCT02321540; NCT02178722; NCT02321540; NCT02403271) and some clinical trials in Phase I (NCT01862081); Phase II (NCT01306045; NCT02289833); Phase III (NCT02134015) at unknown locations are going on HER2 as a target mentioned in Tables 11 and 12 [59].

\section{KRAS}

RAS genes identified as of three types: (1) KRAS (homologous to the oncogene from the Kirsten rat sarcoma virus), (2) homologous to the oncogene from the Harvey rat sarcoma virus, and (3) NRAS (first isolated from a human neuroblastoma). The different RAS genes are highly homologous but functionally distinct; the degree of redundancy remains a topic of investigation. RAS proteins, small
GTPases which cycle between an active guanosine triphosphate (GTP)-bound and inactive guanosine diphosphate (GDP)-bound forms. RAS proteins are a central downstream regulator of growth factor receptor signaling and consequently are critical for cell proliferation, differentiation, and cell survival. RAS can activate several downstream pathways which include the PI3K-AKT-mTOR and the RAS-RAF-MEK-ERK pathway, which involved in cell survival and cell proliferation (Fig. 1) [62]. RAS implicated in the pathogenesis of several cancers. Activating mutations in the RAS gene activate the RAS GTPase, even in the absence of growth factor signaling [62]. KRAS mutations are especially not unusual in colon cancer, lung cancer, and pancreatic cancer [62].

\section{Interventions for KRAS mutations}

Lung adenocarcinomas in addition harbors activating mutations in the downstream GTPase, KRAS, and mutations in EGFR and KRAS appear to be mutually exclusive. As such, no targeted therapies are available for KRAS-mutant non-small-cell lung cancer (NSCLC) even though it is not clear that KRAS mutational status is a predictor of efficacy $[7,63]$.

Supporting targeted therapies against KRAS comes from several studies such as:

- Sorafenib, used as second-line or beyond and KRAS/BRAF marker group who treated with sorafenib had a disease control rate with the stability of illness in patients [64].

- Erlotinib and Gefitinib, as per study Pao et al., patients with mutations in EGFR and KRAS in NSCLC screened to gefitinib or erlotinib. But, the results showed that mutations in KRAS-associated with a lack of sensitivity to either drug [65].

- Trametinib, may also have activity in NSCLC, in a phase II trial, KRAS mutant NSCLC patients randomly assigned to either trametinib or docetaxel as second-line therapy. There were no statistically significant differences between KRAS mutant and KRAS wild-type regarding progression-free survival (PFS) or overall survival (OS). In another phase II trial, patients with advanced NSCLC patients analyzed by KRAS status treated with docetaxel plus trametinib and response rates of approximately less percentage regardless of genotype. In another parallel study, treated cancer patients with pemetrexed plus trametinib also showed fewer response rates regardless of KRAS status as well [63].

- $\quad$ Selumetinib, is an inhibitor of MEK1/MEK2 and downstream regulator of KRAS, with preclinical evidence of synergistic activity with docetaxel in KRAS-mutant cancers [66].

Table 11: In USA, clinical trials targeted as HER2 in different phases are going on mentioned below [58]

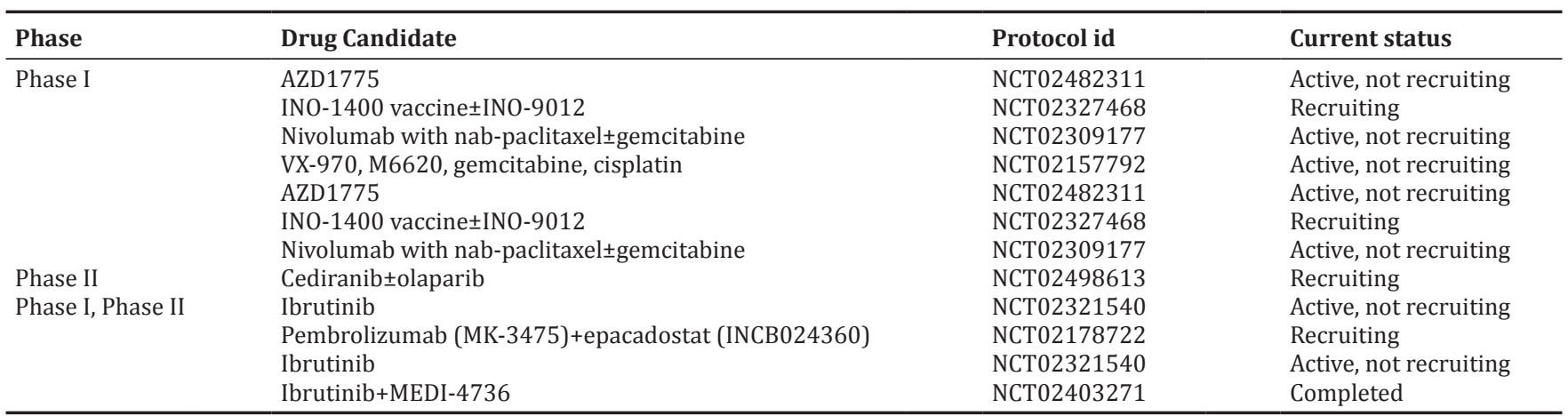

Table 12: Some clinical trials targeted as HER2 in different phases at unknown locations are going on mentioned below [58]

\begin{tabular}{lll}
\hline Phase & Drug candidate & Protocol id \\
\hline Phase I & GDC-0032+docetaxel or paclitaxel & NCT01862081 \\
Phase II & Targeted enzyme inhibitor therapy & NCT01306045 \\
& Trastuzumab emtansine & NCT02289833 \\
Phase III & Patritumab \pm erlotinib & NCT02134015 \\
\hline
\end{tabular}


Clinical trials on KRAS

In the USA, nine clinical trials in Phase I (NCT02071862; NCT01912625; NCT01911507; NCT01859026); Phase II (NCT02047344; NCT01829217; NCT01752400; NCT02642042); Phase III (NCT02152631) and some clinical trials at unknown locations in Phase II (NCT02258607; NCT01988896); Phase II (NCT01877083; NCT01306045); no specified Phase (NCT00900328) are going on KRAS as a target mentioned in Tables 13 and 14 [62].

\section{MEK1 - (MAP2K1)}

MEK1 is the principal mediator of the MAP kinase signaling pathway. It is a receptor serine-threonine protein kinase, and it involved in many cellular processes, including cell proliferation, differentiation, and transcriptional regulation [67].

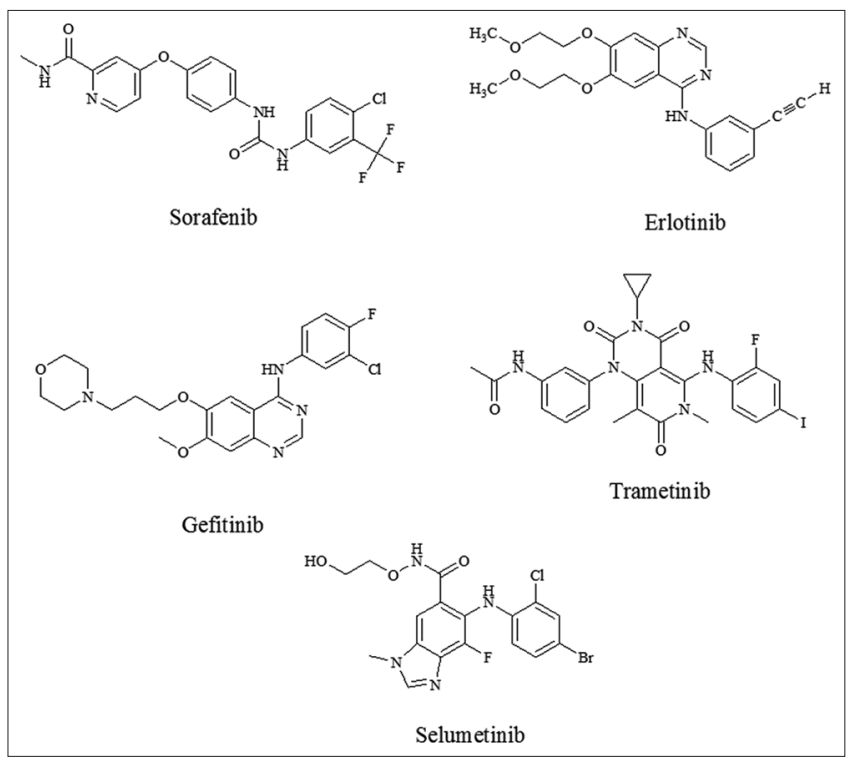

\section{INTERVENTIONS FOR THE MEK1 MUTATIONS}

- Selumetinib, is an oral, MEK1/MEK2 kinases selective inhibitor, and using preclinical data of KRAS mutant NSCLC xenograft models, indicated significant inhibition of tumor growth by selumetinib [68].

- Trametinib, is also an oral, MEK1/MEK2 kinases selective inhibitor, and it revealed activity in cell line and xenograft models and RAS mutant models [68].

\section{Clinical trials on MEK1}

No clinical trials found for the MEK1 target.

\section{NRAS - sub-type RAS gene family}

The different types are of RAS genes functionally distinct but highly homologous. It is a subtype of RAS family and first isolated from a human neuroblastoma. RAS proteins are small GTPases, which cycle between active GTP-bound and inactive GDP-bound forms. These proteins are central mediators downstream of growth factor receptor signaling and therefore are crucial to cell proliferation, differentiation and cell survival. RAS downregulate PI3K-AKT-mTOR and RAS-RAFMEK-ERK pathway, involved in cell survival and cell proliferation (Fig. 1) [69]. NRAS mutations most commonly in melanoma, myeloid leukaemias, and thyroid carcinoma, hepatocellular carcinoma [69].

\section{Interventions for the NRAS mutations}

- Binimetinib, showed robust activity specifically in NRAS-mutant melanoma and an allosteric MEK1/2 inhibitor [70].

- Selumetinib, is a farnesyltransferase inhibitor (FTI) which is a second-generation inhibitor of MEK1/2 and also having potent inhibition of both RAS and RAF-mutant cancers. Further clinical trials of FTIs in melanoma are not ongoing [70].

- Trametinib, is an MEK1/2 allosteric inhibitor.

- Sorafenib is an inhibitor of a multitargeted kinase and tivantinib, a MET inhibitor, also an active against NRAS-mutant melanoma [70].

- SCH772984, was efficacious in cell lines with NRAS, KRAS, and BRAF mutations as well as in models of BRAF inhibitor-resistant melanoma [70].

- Heat shock protein-90, may have a role in the single agent, or combination therapy showed promising activity in NRAS-mutant melanoma inhibition [70].

- Immune-based therapies, such as high-dose interleukin-2, ipilimumab is a monoclonal antibody to cytotoxic T lymphocyte antigen-4 (CTLA4), agents targeting programmed cell death-1 receptor and its ligand (PD-1/PDL-1) Nivolumab (BMS-936558) and pembrolizumab (MK-3475) are some other options for the treatment in NRAS mutations [70].

\section{Clinical trials on NRAS}

Some clinical trials in Phase II (NCT01306045) at unknown locations are going on NRAS as a target is mentioned in Table 15 [69].

\section{PIK3CA}

PI3K are a family of lipid kinases concerned in a lot of cellular processes, along with cell growth, differentiation, proliferation, motility, and survival. PI3K is a heterodimer composed of 2 subunits $110 \mathrm{kDa}$

Table 13: In USA, clinical trials targeted as KRAS in different phases mentioned below [61]

\begin{tabular}{lll}
\hline Phase & Drug candidate & Protocol id \\
\hline Phase I & Glutaminase inhibitor CB-839 and Pac-CB & NCT02071862 \\
& Trametinib, combination chemotherapy, and radiation therapy & NCT01912625 \\
& INC280 and erlotinib hydrochloride & NCT01911507 \\
& MEK162 and erlotinib hydrochloride & NCT01859026 \\
Phase II & Antroquinonol & NCT02047344 \\
& Sunitinib malate & NCT01829217 \\
& AUY922 & NCT01752400 \\
Phase III & Trametinib and docetaxel & NCT02642042 \\
\hline
\end{tabular}

Table 14: Some clinical trials targeted as KRAS in different phases at unknown locations mentioned as follows [61]

\begin{tabular}{llll}
\hline Phase & Drug candidate & Protocol id & Current status \\
\hline Phase I & Momelotinib+trametinib & NCT02258607 & Terminated \\
& Atezolizumab+cobimetinib & NCT01988896 & Active, not recruiting \\
Phase II & Lenvatinib (E7080) & NCT01877083 & Active, not recruiting \\
& Targeted enzyme inhibitor & NCT01306045 & NCT00900328 \\
No phase specified & Laboratory biomarker analysis & Completed & \\
\hline
\end{tabular}


Table 15: Some clinical trials targeted as NRAS in different phases at unknown locations mentioned as follows [68]

\begin{tabular}{llc}
\hline Phase & Drug candidate & Protocol id \\
& & Current \\
\hline Phase II & $\begin{array}{l}\text { Targeted enzyme inhibitor therapy (AZD6244, MK-2206, } \\
\text { Lapatinib) }\end{array}$ & NCT01306045 \\
\hline
\end{tabular}

catalytic subunit and $85 \mathrm{kDa}$ regulatory subunit (p85). The PIK3CA gene also encodes $\mathrm{p} 110 \alpha$, one of the catalytic subunits [71]. PI3K converted PI (4,5) P2 (Phosphatidylinositol 4,5-bisphosphate) to PI $(3,4,5)$ P3 (Phosphatidylinositol [3,4,5]-trisphosphate) on the inner side of the cell membrane. PI $(3,4,5)$ P3 recruits crucial downstream signaling proteins, inclusive AKT, to the cell membrane resulting in increased activity of these proteins [71]. PIK3CA mutation implicated in the pathogenesis of numerous cancers, including colon cancer, gliomas, breast cancer, gastric cancer, endometrial cancer, and lung cancer [71]

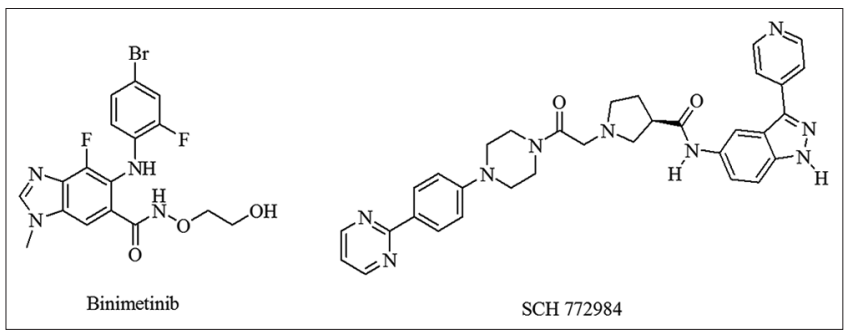

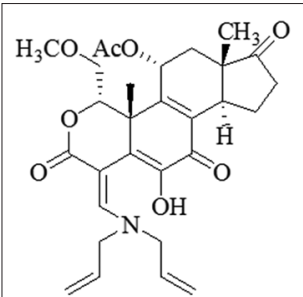

PX-866<smiles>Nc1cc(C(F)(F)F)c(-c2cc(N3CCOCC3)nc(N3CCOCC3)n2)cn1</smiles>

NVP-BKM120<smiles>Cc1nc(NC(=O)N2CCC[C@H]2C(N)=O)sc1-c1ccnc(C(C)(C)C(F)(F)F)c1</smiles>

NYP-BYL 719

\section{Interventions for the PIK3CA mutations}

Currently, several inhibitors directed against PI3K are being clinically evaluated for NSCLC treatment as follows:

- PX-866, is a pan class-I PI3K inhibitor that binds PI3K irreversibly and developed from biologically stable semisynthetic viridins [72].

- NVP-BKM120, GDC-0941, XL147, are specific pan-class I PI3K inhibitors [72]

- BAY 80-6946, represents a novel, highly selective and potent panclass I PI3K inhibitor, which has preferential activity against the $\mathrm{p} 110 \alpha$ and $\mathrm{p} 110 \delta$ isoforms, compared with $\mathrm{p} 110 \alpha$ and $\mathrm{p} 110 \gamma$ [72].

- NVP-BYL719, is an inhibitor of a p111 $\alpha$ catalytic subunit of PI3K, GSK-2636771 is a p110 $\beta$ selective inhibitor, GDC-0032 is a potent next-generation PI3K inhibitor [72].

\section{Clinical trials on PIK3CA}

In the USA, eight clinical trials in Phase 0 (NCT02357836); Phase I (NCT02079636; NCT01920061; NCT01859026); Phase II (NCT02642042; NCT01827384) and some clinical trials in Phase I, II (NCT02393209) at unknown locations are going on PIK3CA as a target mentioned in Tables 16 and 17 [71].
RET

The RET gene belongs to the RET family of RTKs and positioned on chromosome 10 and encodes a RTK. This gene plays a significant role in neural crest development. Binding of its ligands, the glial cell line-derived neurotrophic factor family of extracellular signaling molecules, regulates receptor phosphorylation and activation. After phosphorylation RET-activated, then its substrates, resulting in activation of multiple downstream cellular pathways (Fig. 6) [73]. Genomic alterations in RET found in several different types of cancer. Activating point mutations in RET can give rise to the hereditary cancer syndrome, multiple endocrine neoplasias 2 (MEN2). Somatic point mutations in RET also associated with sporadic medullary thyroid cancer. Oncogenic kinase fusions involving the RET gene found in $~ 1 \%$ of NSCLC [73].

\section{Interventions for the RET mutations}

- Sorafenib, has potent anti-RET activity and also a multi-kinase inhibitor. Since, sorafenib did not show dramatic results, the study suggesting for testing of other RET inhibitors in the treatment of RET fusion-positive NSCLC [74].

- Sunitinib, is a RET tyrosine kinase inhibitor, and preclinical data of sunitinib suggest that it can play an important role in the clinical development of NSCLC treatment $[74,75]$.

- Cabozantinib, in patients with RET fusion-positive NSCLC cancer, provides new drug treatment paradigm in lung cancers. Toxicities related to cabozantinib are manageable and RET fusion investigations [76].

- Vandetanib, is a RET inhibitor in NSCLC patients [77].

In unselected NSCLC patient, clinical trial conducted, but all these drugs did not show a survival benefit in RET mutations in NSCLC.

\section{Clinical trials on RET}

In the USA, eight clinical trials in Phase I (NCT02608385; NCT02409108); Phase II (NCT02544633; NCT02498613; NCT02426658; NCT02312622; NCT02132598; NCT02045446; NCT01935336; NCT01928576;

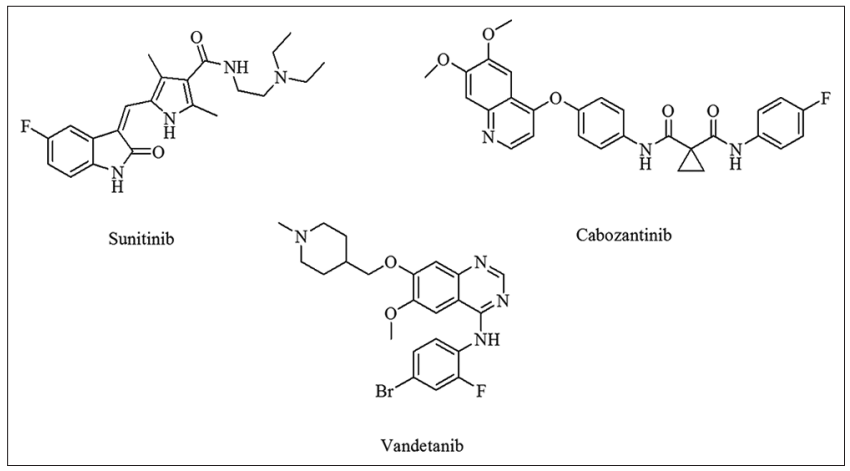

NCT01829217; NCT01822496; NCT01813734; NCT01702844; NCT01639508); Phase III (NCT02595944); no specified Phase (NCT02299141; NCT02194738; NCT02193152) and some clinical trials in Phase I (NCT01839955); Phase II (NCT02485652; NCT01877083; NCT01306045); Phase III (NCT02296125); Phase IV (NCT02151149) at unknown locations are going on RET as a target mentioned in Tables 18 and 19 [73]. 
Table 16: In USA, clinical trials targeted as PIK3CA in different phases mentioned below [70]

\begin{tabular}{|c|c|c|c|}
\hline Phase & Drug candidate & Protocol id & Current status \\
\hline Phase 0 & Itraconazole & NCT02357836 & Recruiting \\
\hline \multirow[t]{3}{*}{ Phase I } & Abemaciclib (LY2835219) & NCT02079636 & Recruiting \\
\hline & PF-05212384+docetaxel, cisplatin, dacomitinib & NCT01920061 & Recruiting \\
\hline & MEK-162 and erlotinib hydrochloride & NCT01859026 & Recruiting \\
\hline \multirow[t]{2}{*}{ Phase II } & Trametinib and docetaxel & NCT02642042 & Recruiting \\
\hline & Molecular profiling-based targeted therapy & NCT01827384 & Suspended \\
\hline
\end{tabular}

Table 17: Some clinical trials targeted as PIK3CA in different phases at unknown places as mentioned below [70]

\begin{tabular}{llll}
\hline Phase & Drug candidate & Protocol id & Current status \\
\hline Phase I, Phase II & Docetaxel \pm MLN1117 & NCT02393209 & Terminated \\
\hline
\end{tabular}

Table 18: In USA, clinical trials targeted as RET in different phases mentioned below [78]

\begin{tabular}{|c|c|c|c|}
\hline Phase & Drug candidate & Protocol id & Current status \\
\hline \multirow[t]{2}{*}{ Phase I } & Pembrolizumab and stereotactic body radiation therapy & NCT02608385 & Recruiting \\
\hline & Exatherm total-body hyperthermia & NCT02409108 & Recruiting \\
\hline \multirow[t]{11}{*}{ Phase II } & MGCD265 & NCT02544633 & Recruiting \\
\hline & Cediranib \pm Olaparib & NCT02498613 & Recruiting \\
\hline & Pemetrexed disodium & NCT02426658 & Recruiting \\
\hline & Pegylated irinotecan NKTR-102 & NCT02312622 & Recruiting \\
\hline & Cabozantinib-s-malate & NCT02132598 & Recruiting \\
\hline & Ponatinib hydrochloride & NCT01935336 & Recruiting \\
\hline & Azacitidine, entinostat, and nivolumab or nivolumab & NCT01928576 & Recruiting \\
\hline & Sunitinib malate & NCT01829217 & Active, not recruiting \\
\hline & $\begin{array}{l}\text { Erlotinib hydrochloride or crizotinib and chemoradiation } \\
\text { therapy }\end{array}$ & NCT01822496 & Active, not recruiting \\
\hline & Ponatinib hydrochloride & NCT01813734 & Active, not recruiting \\
\hline & Paclitaxel albumin-stabilized nanoparticle formulation & NCT01702844 & Active, not recruiting \\
\hline Phase III & Nivolumab after surgery+chemotherapy & NCT02595944 & Recruiting \\
\hline \multirow[t]{3}{*}{ No phase specified } & Nintedanib & NCT02299141 & Recruiting \\
\hline & Genetic testing & NCT02194738 & Recruiting \\
\hline & Pazopanib hydrochloride & NCT02193152 & Recruiting \\
\hline
\end{tabular}

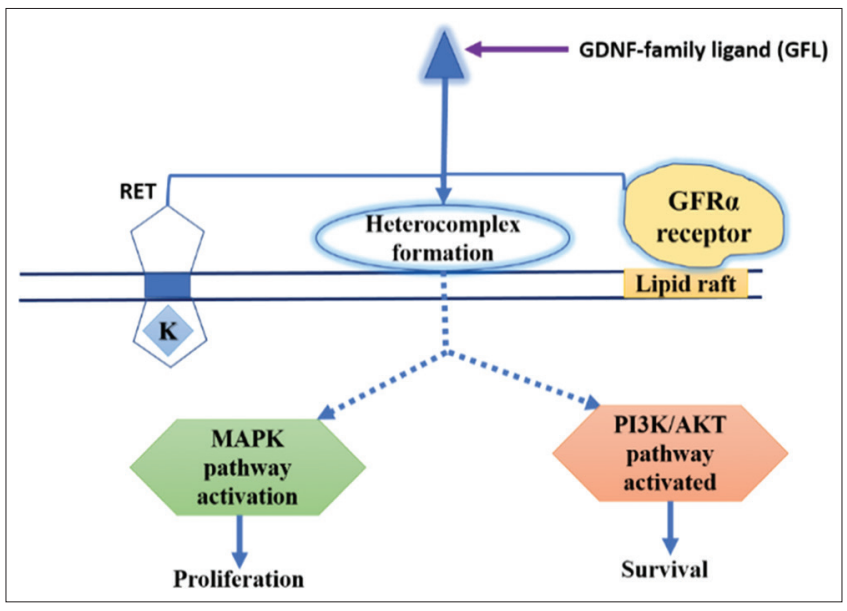

Fig. 6: Signaling pathway rearranged during transfection (RET). RET activation involves binding of glial cell line derived neurotrophic factor-family ligands as well as interaction with GFR alpha receptors, resulting in activation of intracellular MAPK and PI3K pathways. The letter " $K$ " within the schema denotes the tyrosine kinase domain.

\section{MET}

The MET gene (MNNG-HOS transforming gene) present on chromosome 7 and encodes a RTK which is belonging to the MET/RON family of
RTKs. After, binding of its ligand called as hepatocyte growth factor (HGF; also known as scatter factor), which induces a conformational change within the MET receptor that allows receptor phosphorylation as well as activation. After phosphorylation of its substrates, resulting in activation of multiple downstream pathways within the cell after MET activation, including the PI3K-AKT-mTOR pathway, which is involved in cell survival, and the RAS-RAF-MEK-ERK pathway, which is involved in cell proliferation (Fig. 7). In the context of malignancy, aberrant signaling through the MET receptor promotes pleiotropic effects, including growth, survival, invasion, migration, angiogenesis, and metastasis [78].

The MET receptor and its ligand HGF were mentioned to be aberrantly activated in many human cancers. Germline mutations in MET tyrosine kinase domain, arise in all of the hereditary papillary renal cell carcinomas, Somatic mutations in MET found in $10-15 \%$ of sporadic papillary renal cell carcinoma. Mutations in MET reported at low frequencies in head and neck squamous cell carcinoma, childhood hepatocellular carcinoma, NSCLC, and SCLC. Amplification of MET reported in gastric cancer, esophageal cancer, colorectal cancer, gliomas, clear cell ovarian cancer, and NSCLC [78].

\section{Interventions for the MET mutations}

- Tivantinib, is dual EGFR and MET inhibitors, with erlotinib and tivantinib, respectively, tested in non-squamous NSCLC within the awful lot anticipated global phase III trial MARQUEE, after Phase II data [17]. 
Table 19: Clinical trials targeted as RET in different phases at unknown locations mentioned below [78]

\begin{tabular}{lll}
\hline Phase & Drug candidate & Protocol id \\
\hline Phase I & Erlotinib hydrochloride and quinacrine dihydrochloride & NCT01839955 \\
Phase II & HM61713 (BI-1482694) & NCT02485652 \\
& Lenvatinib (E7080) & NCT01877083 \\
Phase III & Targeted enzyme inhibitor therapy & NCT01306045 \\
Phase IV & AZD9291 vs gefitinib or erlotinib & NCT02296125 \\
Active, not recruiting & Recruiting recruiting \\
Act02151149 & Completed \\
\hline
\end{tabular}

RET: Rearranged during transfection

Table 20: In USA, clinical trials targeted as MET in different phases mentioned below [77]

\begin{tabular}{|c|c|c|c|}
\hline Phase & Drug candidate & Protocol id & Current status \\
\hline Phase I & INC280+erlotinib hydrochloride & NCT01911507 & Recruiting \\
\hline \multirow[t]{5}{*}{ Phase II } & MGCD265 & NCT02544633 & Recruiting \\
\hline & $\begin{array}{l}\text { Chemotherapy and radiation therapy } \pm \text { metformin } \\
\text { hydrochloride }\end{array}$ & NCT02186847 & Active, not recruiting \\
\hline & Cabozantinib-s-malate & NCT02132598 & Recruiting \\
\hline & Cabozantinib-s-malate & NCT01639508 & Recruiting \\
\hline & Cabozantinib-s-malate & NCT01588821 & Active, not recruiting \\
\hline Phase II, Phase III & Biomarker-targeted second-line therapy & NCT02154490 & Recruiting \\
\hline
\end{tabular}

Table 21: Some clinical trials targeted as MET in different phases at unknown locations as mentioned below [77]

\begin{tabular}{llll}
\hline Phase & Drug candidate & Protocol id \\
\hline Phase II & Pemetrexed disodium and carboplatin or cisplatin \pm erlotinib hydrochloride & NCT01928160 & Withdrawn \\
No phase specified & Pilot study of c-MET and p53 expression & NCT00900328 & Completed \\
\hline
\end{tabular}

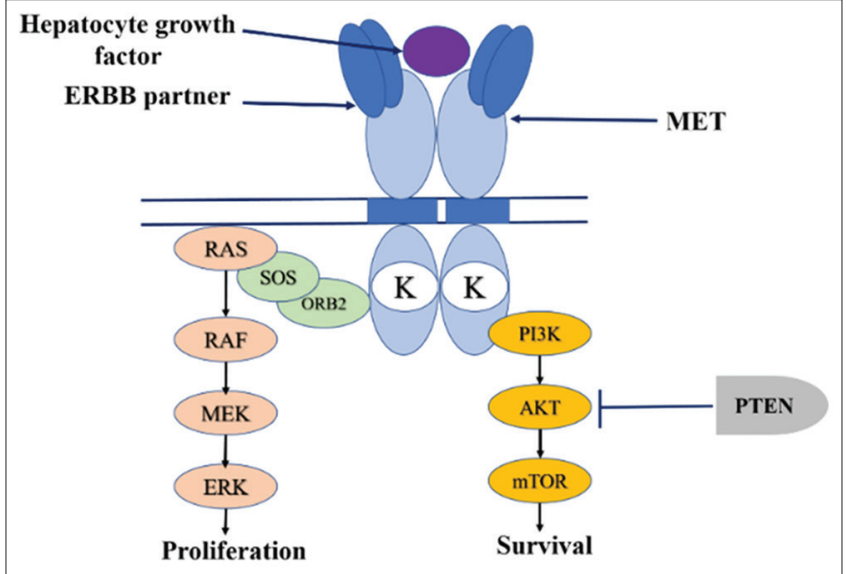

Fig. 7: Cell signaling pathway for MET. Growth factor binding to MET results in activation of the MAPK signaling pathway (RASRAF-MEK-ERK) and the phosphatidylinositol 3-kinase (PI3K) pathway (PI3K-AKT-mTOR). The letter "K" within the schema denotes the tyrosine kinase domain.

- Onartuzumab, is a monoclonal antibody against MET showed treatment in a Phase II trial [17].

\section{Clinical trials on MET}

In the USA, eight clinical trials in Phase I (NCT01911507); Phase II (NCT02544633; NCT02186847; NCT02132598; NCT01639508; NCT01588821; NCT01553942); Phase II, III (NCT02154490) and some clinical trials Phase II (NCT01928160); no specified Phase (NCT00900328) at unknown locations are going on MET as a target mentioned in Tables 20 and Table 21 [78].

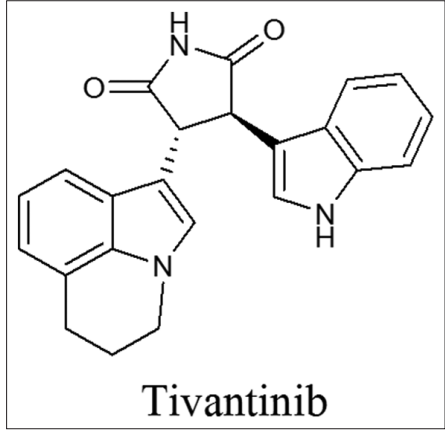

\section{CONCLUSION}

The aim of this review is to analyze the current trends in the targets due to the genetic modulation with in the current updates of interventions and clinical trials. The treatment of genetic modulated NSCLC and the current scenario of drug discovery have been touched in a comprehensive way. In future, it will be an important discussion point among the all drug scientist. It is also evident that the intervention of NSCLC just in regard to the changes in genes or genetic mutations that is varied and continues to be studied and analyzed to most benefit treatment of NSCLC patients and society as a whole.

\section{AUTHOR CONTRIBUTIONS}

All authors have participated in (a) conception and design; (b) drafting the article or revising it critically for important intellectual content; and (c) approval of the final version.

\section{CONFLICT OF INTEREST}

The authors have no affiliation with any organization with a direct or indirect financial interest in the subject matter discussed in the manuscript. 


\section{REFERENCES}

1. Rahman M, Islam SN. Effect of serum antioxidants (Vitamin E, C and A) in lung cancer patients. Int J Pharm Pharm Sci 2014;6:7-9.

2. Pfizer. Lung Cancer Fact Sheet. Pfizer; 2012.

3. Molina JR, Yang P, Cassivi SD, Schild SE, Adjei AA. Non-small cell lung cancer: Epidemiology, risk factors, treatment, and survivorship Mayo Clin Proc 2008;83:584-94.

4. American Cancer Society. Lung Cancer (Non-Small Cell) What is nonsmall cell lung cancer? Am Cancer Soc 2016;1:1-75.

5. Zappa C, Mousa SA. Non-small cell lung cancer: Current treatment and future advances. Transl Lung Cancer Res 2016;5:288-300.

6. Gupta M, Dahiya J, Marwaha RK, Dureja H. Therapies in cancer treatment: An overview. Int J Pharm Pharm Sci 2015;7:1-9.

7. Karachaliou N, Mayo C, Costa C, Magrí I, Gimenez-Capitan A, Molina-Vila MA, et al. KRAS mutations in lung cancer. Clin Lung Cancer 2013;14:205-14

8. Wang J, Song J, Gao Z, Huo X, Zhang Y, Wang W, et al. Analysis of gene expression profiles of non-small cell lung cancer at different stages reveals significantly altered biological functions and candidate genes. Oncol Rep 2017;37:1736-46.

9. Thunnissen E, van der Oord K, den Bakker M. Prognostic and predictive biomarkers in lung cancer. A review. Virchows Arch 2014;464:347-58.

10. Johnson JL, Pillai S, Chellappan SP. Genetic and biochemical alterations in non-small cell lung cancer. Biochem Res Int 2012;2012:940405.

11. Risch A, Plass C. Lung cancer epigenetics and genetics. Int J Cancer 2008;123:1-7.

12. Lazarus DR, Ost DE. How and when to use genetic markers for nonsmall cell lung cancer. Curr Opin Pulm Med 2013;19:331-9.

13. Zhu QG, Zhang SM, Ding XX, He B, Zhang HQ. Driver genes in nonsmall cell lung cancer: Characteristics, detection methods, and targeted therapies. Oncotarget 2017;8:57680-92.

14. Black RC, Khurshid H. NSCLC: An update of driver mutations, their role in pathogenesis and clinical significance. R I Med J (2013) 2015;98:25-8

15. Nan X, Xie C, Yu X, Liu J. EGFR TKI as first-line treatment for patients with advanced EGFR mutation-positive non-small-cell lung cancer. Oncotarget 2017;8:75712-26.

16. Lovly C, Horn L, Pao W. EGFR in non-small cell lung cancer (NSCLC). Cancer Genome 2015;1:1.

17. Chan BA, Hughes BG. Targeted therapy for non-small cell lung cancer: Current standards and the promise of the future. Transl Lung Cancer Res 2015;4:36-54

18. Zappa C, Mousa SA. Non-small cell lung cancer: current treatment and future advances. Transl Lung Cancer Res 2016;5:288-300.

19. Seshacharyulu P, Ponnusamy MP, Haridas D, Jain M, Ganti AK, Batra SK, et al. Targeting the EGFR signaling pathway in cancer therapy. Expert Opin Ther Targets 2012;16:15-31.

20. Bittner N, Ostoros G, Géczi L. New treatment options for lung adenocarcinoma-in view of molecular background. Pathol Oncol Res 2014;20:11-25.

21. Shi Y, Zhang L, Liu X, Zhou C, Zhang L, Zhang S, et al. Icotinib versus gefitinib in previously treated advanced non-small-cell lung cancer (ICOGEN): A randomised, double-blind phase 3 non-inferiority trial. Lancet Oncol 2013;14:953-61.

22. Lemmon MA, Schlessinger J. Cell signaling by receptor tyrosine kinases. Cell 2010;141:1117-34.

23. Masters GA, Temin S, Azzoli CG, Giaccone G, Baker S, Brahmer JR, et al. Systemic therapy for stage IV non-small-cell lung cancer: American society of clinical oncology clinical practice guideline update. J Clin Oncol 2015;33:3488-515.

24. Douillard JY, Ostoros G, Cobo M, Ciuleanu T, McCormack R, Webster A, et al. First-line gefitinib in caucasian EGFR mutationpositive NSCLC patients: A phase-IV, open-label, single-arm study. $\mathrm{Br}$ J Cancer 2014;110:55-62.

25. Pirker R, Filipits M. Cetuximab in non-small-cell lung cancer. Transl Lung Cancer Res 2012;1:54-60.

26. Sgambato A, Casaluce F, Maione P, Rossi A, Ciardiello F, Gridelli C, et al. Cetuximab in advanced non-small cell lung cancer (NSCLC): The showdown? J Thorac Dis 2014;6:578-80.

27. Jain P, Khanal R, Sharma A, Yan F, Sharma N. Afatinib and lung cancer. Expert Rev Anticancer Ther 2014;14:1391-406.

28. Sequist LV, Besse B, Lynch TJ, Miller VA, Wong KK, Gitlitz B, et al. Neratinib, an irreversible pan-erbB receptor tyrosine kinase inhibitor: Results of a phase II trial in patients with advanced non-small-cell lung cancer. J Clin Oncol 2010;28:3076-83.

29. Simon GR, Garrett CR, Olson SC, Langevin M, Eiseman IA, Mahany JJ, et al. Increased bioavailability of intravenous versus oral CI-1033, a pan erbB tyrosine kinase inhibitor: Results of a phase I pharmacokinetic study. Clin Cancer Res 2006;12:4645-51.

30. Wang S, Song Y, Yan F, Liu D. Mechanisms of resistance to thirdgeneration EGFR tyrosine kinase inhibitors. Front Med 2016;10:383-8.

31. Liao BC, Lin CC, Yang JC. Second and third-generation epidermal growth factor receptor tyrosine kinase inhibitors in advanced nonsmall cell lung cancer. Curr Opin Oncol 2015;27:94-101.

32. Barnes TA, O'Kane GM, Vincent MD, Leighl NB. Third-generation tyrosine kinase inhibitors targeting epidermal growth factor receptor mutations in non-small cell lung cancer. Front Oncol 2017;7:113.

33. Narita SN, Kanda S. Overcoming resistance to third-generation epidermal growth factor receptor tyrosine kinase inhibitor in non-small cell lung cancer. Transl Cancer Res 2017;6:S1187-90.

34. Lee MW, Kim DS, Lee JH, Lee BS, Lee SH, Jung HL, et al. Roles of AKT1 and AKT2 in non-small cell lung cancer cell survival, growth, and migration. Cancer Sci 2011;102:1822-8.

35. Lovly C, Horn L, Pao W. AKT1 in non-small cell lung cancer (NSCLC). Cancer Genome 2015;1:1

36. Vijayalakshmi R, Krishnamurthy A. Targetable "driver" mutations in non small cell lung cancer. Indian J Surg Oncol 2011;2:178-88.

37. Greulich H. The genomics of lung adenocarcinoma: Opportunities for targeted therapies. Genes Cancer 2010;1:1200-10.

38. LoRusso PM. Inhibition of the PI3K/AKT/mTOR pathway in solid tumors. J Clin Oncol 2016;34:3803-15.

39. Lin J, Sampath D, Nannini MA, Lee BB, Degtyarev M, Oeh J, et al. Targeting activated akt with GDC-0068, a novel selective akt inhibitor that is efficacious in multiple tumor models. Clin Cancer Res 2013;19:1760-72.

40. Pal SK, Reckamp K, Yu H, Figlin RA. Akt inhibitors in clinical development for the treatment of cancer. Expert Opin Investig Drugs 2010;19:1355-66.

41. Lovly C, Horn L, Pao W. BRAF in non-small cell lung cancer (NSCLC). Cancer Genome 2015;1:1.

42. de Langen AJ, Smit EF. Therapeutic approach to treating patients with BRAF-mutant lung cancer: Latest evidence and clinical implications. Ther Adv Med Oncol 2017;9:46-58.

43. Baik CS, Myall NJ, Wakelee HA. TargetingBRAF-mutant non-small cell lung cancer: From molecular profiling to rationally designed therapy. Oncologist 2017;22:786-96

44. Caparica R, de Castro G Jr., Gil-Bazo I, Caglevic C, Calogero R, Giallombardo $\mathrm{M}$, et al. BRAF mutations in non-small cell lung cancer: Has finally janus opened the door? Crit Rev Oncol Hematol 2016;101:32-9

45. Ding X, Zhang Z, Jiang T, Li X, Zhao C, Su B, et al. Clinicopathologic characteristics and outcomes of chinese patients with non-small-cell lung cancer and BRAF mutation. Cancer Med 2017;6:555-62.

46. Luk PP, Yu B, Ng CC, Mercorella B, Selinger C, Lum T, et al. BRAF mutations in non-small cell lung cancer. Transl Lung Cancer Res 2015;4:142-8

47. Hyman DM, Puzanov I, Subbiah V, Faris JE, Chau I, Blay JY, et al. Vemurafenib in multiple nonmelanoma cancers with BRAF V600 mutations. N Engl J Med 2015;373:726-36.

48. Planchard D, Kim TM, Mazieres J, Quoix E, Riely G, Barlesi F, et al. Dabrafenib in BRAF V600E-mutant advanced non-small cell lung cancer: An open-label, single arm, multicenter, phase 2 trial. Lancet Oncol 2016;17:642-50.

49. Lovly C, Horn L, Pao W. ALK in non-small cell lung cancer (NSCLC). Cancer Genome 2015;1:1.

50. Katayama R, Lovly CM, Shaw AT. Therapeutic targeting of anaplastic lymphoma kinase in lung cancer: A paradigm for precision cancer medicine. Clin Cancer Res 2015;21:2227-35.

51. Qin A, Gadgeel S. The current landscape of anaplastic lymphoma kinase (ALK) in non-small cell lung cancer: Emerging treatment paradigms and future directions. Target Oncol 2017;12:709-18.

52. Iams WT, Lovly CM. Anaplastic lymphoma kinase as a therapeutic target in non-small cell lung cancer. Cancer J 2015;21:378-82.

53. Sequist LV, Neal JW. Personalized, Genotype-Directed Therapy for Advanced Non-Small Cell Lung Cancer. Uptodate; 2016. p. 1

54. Lovly C, Horn L, Pao W. ROS1 in non-small cell lung cancer (NSCLC). Cancer Genome 2015;1:1.

55. Bubendorf L, Büttner R, Al-Dayel F, Dietel M, Elmberger G, Kerr K, et al. Testing for ROS1 in non-small cell lung cancer: A review with recommendations. Virchows Arch 2016;469:489-503.

56. Shaw AT, Ou SH, Bang YJ, Camidge DR, Solomon BJ, Salgia R, et al. Crizotinib in ROS1-rearranged non-small-cell lung cancer. N Engl J Med 2014;371:1963-71. 
57. Mazières J, Rouvière D, Milia JD, Filleron T, Zalcman G, Biondani P, et al. Crizotinib therapy for advanced lung adenocarcinoma and a ROS1 rearrangement: Results from the EUROS1 cohort. J Clin Oncol 2015;33:992-9.

58. FDA. FDA Expands Use of Advanced therapy for Non-Small Cell Lung Cancer. Am Assoc Cancer Res 2016;1:1.

59. Lovly C, Shi GC, Watson L, Horn P, Pohlmann LG. HER2 (ERBB2) in non-small cell lung cancer (NSCLC). Cancer Genome 2015;1:1.

60. Peters S, Zimmermann S. Targeted therapy in NSCLC driven by HER2 insertions. Transl Lung Cancer Res 2014;3:84-8.

61. Garrido-Castro AC, Felip E. HER2 driven non-small cell lung cancer (NSCLC): Potential therapeutic approaches. Transl Lung Cancer Res 2013;2:122-7.

62. Lovly C, Horn L, Pao W. KRAS in non-small cell lung cancer (NSCLC). Cancer Genome 2015;1:1.

63. Blumenschein GR Jr., Smit EF, Planchard D, Kim DW, Cadranel J, De Pas T, et al. A randomized phase II study of the MEK1/MEK2 inhibitor trametinib (GSK1120212) compared with docetaxel in KRAS-mutant advanced non-small-cell lung cancer (NSCLC) †. Ann Oncol 2015;26:894-901.

64. Kim ES, Herbst RS, Wistuba II, Lee JJ Jr., Blumenschein GR, Tsao A, et al. The BATTLE Trial: Personalizing therapy for lung cancer. Cancer Discov 2011;1:44-53.

65. Pao W, Wang TY, Riely GJ, Miller VA, Pan Q, Ladanyi M, et al. KRAS mutations and primary resistance of lung adenocarcinomas to gefitinib or erlotinib. PLoS Med 2005;2:57-61.

66. Jänne PA, Shaw AT, Pereira JR, Jeannin G, Vansteenkiste J, Barrios C, et al. Selumetinib plus docetaxel for KRAS-mutant advanced nonsmall-cell lung cancer: A randomised, multicentre, placebo-controlled, phase 2 study. Lancet Oncol 2013;14:38-47.
67. Lovly C, Horn L, Pao W. MEK1 (MAP2K1) in non-small cell lung cancer (NSCLC). Cancer Genome 2015;1:1.

68. Stinchcombe TE, Johnson GL, Comprehensive L, Hill C, Comprehensive L, Hill C. MEK inhibition in non-small cell lung cancer. Lung Cancer 2014;86:121-5.

69. Lovly C, Horn L, Pao W. NRAS in non-small cell lung cancer (NSCLC). Cancer Genome 2015;1:1.

70. Johnson DB, Puzanov I. Treatment of NRAS-mutant melanoma. Curr Treat Options Oncol 2015;16:15.

71. Lovly C, Horn L, Pao W. PIK3CA in non-small cell lung cancer (NSCLC). Cancer Genome 2015;1:1.

72. Fumarola C, Bonelli MA, Petronini PG, Alfieri RR. Targeting PI3K $\mathrm{AKT} / \mathrm{mTOR}$ pathway in non small cell lung cancer. Biochem Pharmacol 2014;90:197-207.

73. Espinosa AJ. RET in Lung Cancer. Cancer Genome 2015;1:1.

74. Horiike A, Takeuchi K, Uenami T, Kawano Y, Tanimoto A, Kaburaki K, et al. Sorafenib treatment for patients with RET fusion-positive nonsmall cell lung cancer. Lung Cancer 2016;93:43-6.

75. Gridelli C, Maione P, Del Gaizo F, Colantuoni G, Guerriero C, Ferrara C, et al. Sorafenib and sunitinib in the treatment of advanced non-small cell lung cancer. Oncologist 2007;12:191-200.

76. Drilon A, Wang L, Hasanovic A, Suehara Y, Lipson D, Stephens P, et al. Response to cabozantinib in patients with RET fusion-positive lung adenocarcinomas. Cancer Discov 2013;3:630-5.

77. Gautschi O, Zander T, Keller FA, Strobel K, Hirschmann A, Aebi S, et al. A patient with lung adenocarcinoma and RET fusion treated with vandetanib. J Thorac Oncol 2013;8:e43-4.

78. Solomon B. MET in non-small cell lung cancer (NSCLC)-My Cancer Genome. Cancer Genome 2015;1:1.

79. Riely G. RET in lung cancer. Cancer Genome 2013;1:1 